\title{
MicroRNA co-expression networks exhibit increased complexity in pancreatic ductal compared to Vater's papilla adenocarcinoma
}

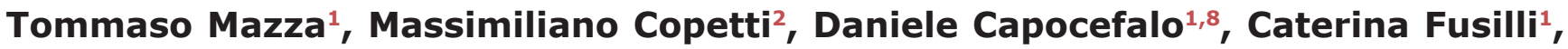 \\ Tommaso Biagini ${ }^{1}$, Massimo Carella ${ }^{3}$, Antonio De Bonis ${ }^{4}$, Nicola Mastrodonato ${ }^{4}$, \\ Ada Piepoli ${ }^{5}$, Valerio Pazienza ${ }^{5}$, Evaristo Maiello', Fabio Francesco di Mola?, \\ Pierluigi di Sebastiano7, Angelo Andriulli ${ }^{5}$ and Francesca Tavano ${ }^{5}$ \\ ${ }^{1}$ Unit of Bioinformatics, Research Hospital, San Giovanni Rotondo 71013, Italy \\ ${ }^{2}$ Unit of Biostatistics, Research Hospital, San Giovanni Rotondo 71013, Italy \\ ${ }^{3}$ Medical Genetics Unit, Research Hospital, San Giovanni Rotondo 71013, Italy \\ ${ }^{4}$ Department of Surgery, Research Hospital, San Giovanni Rotondo 71013, Italy \\ ${ }^{5}$ Division of Gastroenterology and Research Laboratory, San Giovanni Rotondo 71013, Italy \\ ${ }^{6}$ Department of Oncology IRCCS "Casa Sollievo della Sofferenza", Research Hospital, San Giovanni Rotondo 71013, Italy \\ ${ }^{7}$ Division of Surgical Oncology "SS Annunziata" Hospital, Chieti 66100, Italy \\ ${ }^{8}$ Department of Cellular Biotechnologies and Haematology, Sapienza University of Rome, Rome 00161, Italy \\ Correspondence to: Francesca Tavano, email: f.tavano@operapadrepio.it \\ Keywords: microRNA; pancrearic ductal adenocarcinoma; ampullary carcinoma \\ Received: December 06, $2016 \quad$ Accepted: July 11,2017 Published: October 31, 2017 \\ Copyright: Mazza et al. This is an open-access article distributed under the terms of the Creative Commons Attribution License \\ 3.0 (CC BY 3.0), which permits unrestricted use, distribution, and reproduction in any medium, provided the original author and \\ source are credited.
}

\section{ABSTRACT}

MiRNA expression abnormalities in adenocarcinoma arising from pancreatic ductal system (PDAC) and Vater's papilla (PVAC) could be associated with distinctive pathologic features and clinical cancer behaviours. Our previous miRNA expression profiling data on PDAC $(n=9)$ and PVAC $(n=4)$ were revaluated to define differences/ similarities in miRNA expression patterns. Afterwards, in order to uncover target genes and core signalling pathways regulated by specific miRNAs in these two tumour entities, miRNA interaction networks were wired for each tumour entity, and experimentally validated target genes underwent pathways enrichment analysis.

One hundred and one miRNAs were altered, mainly over-expressed, in PDAC samples. Twenty-six miRNAs were deregulated in PVAC samples, where more miRNAs were down-expressed in tumours compared to normal tissues. Four miRNAs were significantly altered in both subgroups of patients, while 27 miRNAs were differentially expressed between PDAC and PVAC.

Although miRNA interaction networks were more complex and dense in PDAC than in PVAC, pathways enrichment analysis uncovered a functional overlapping between PDAC and PVAC. However, shared signalling events were influenced by different miRNA and/or genes in the two tumour entities.

Overall, specific miRNA expression patterns were involved in the regulation of a limited core signalling pathways in the biology landscape of PDAC and PVAC. 


\section{INTRODUCTION}

Pancreatic cancer is one of the most lethal malignancies which ranks fourth among the most prominent causes of cancer-related deaths in Western countries [1]. Although the exocrine pancreas consists of a very small ductal system, $90 \%$ of pancreatic neoplasms are ductal in origin and are known as pancreatic ductal adenocarcinomas (PDAC) [2]. The propensity of PDAC to invade nearby organs and surrounding tissues, as well as the lack of effective means for screening and early detection, contribute to a significant delay in diagnosis, which thus usually occurs only after having reached advanced disease stages [3]. Consequently, the percentage of resectable patients is low and the average survival of these patients is approximately 12 to 20 months, with a high probability of relapse [2-4].

Adenocarcinoma of ampulla of Vater (PVAC) differs from PDAC in prognosis and in how it occurs. Based on its localization, PVAC is usually detected relatively early: the confluence of the common bile duct and the main pancreatic duct at the ampulla are signs that the tumours that arise at this location have the potential to obstruct two major organs and to result in relatively early onset of symptoms, which, most commonly, are biliary obstruction and pancreatitis. This explains why these lesions account for about half of all resectable pancreatic neoplasms, often before disease spreading to lymph nodes, and why PVAC patients have a consistently better survival than patients with PDAC [5-7]. However, the difference in survival is not entirely explained by the lower frequency of lymph node involvement in PVAC. In fact, the survival for nodepositive patients is still better than in PDAC and this difference may be explained by biological, particularly molecular, differences between the two cancer entities.

Recently, microRNAs (miRNAs) have grabbed a wide attention for their pivotal involvement in cancer development [8]. MiRNAs influence various biological processes, including cell proliferation, cell death and stress resistance, mainly by of gene expression [9]. The relationship between the miRNA expression dysregulation and the survival of patients affected by pancreatic adenocarcinoma was already reported in PDAC [10-13]. Conversely, little is known about the potential involvement of miRNAs in the onset and development of PVAC.

Our hypothesis is that several molecular characteristics of PDAC and PVAC, including the miRNA expression abnormalities, could be associated with distinctive pathologic features and clinical behaviours. Overall the aim of this study was to make a comparison between these two tumour entities on the pathogenetic level. For this purpose we defined differences and similarities of miRNA expression patterns of patients with PDAC and PVAC, up to respective target genes and signalling pathways. To this extent, we attempted to unravel the landscape of miRNA expression among PDAC and PVAC using co-expression networks as a proxy for highlighting the main differences between them and their healthy counterpart, as correlation networks are often used as discovery tools [14].

\section{RESULTS}

\section{MiRNAs differentially expressed in tumour versus normal tissues from PDAC and PVAC patients}

One hundred and one miRNAs, out of 1105 miRNAs assayed in our array experiments, were differentially expressed in PDAC tumours, compared to their adjacent normal tissue samples (68 over-expressed and 33 downregulated). MiR-887 was the most significantly altered miRNA $\left(\mathrm{p}=9.21 \times 10^{-5}\right)$, (Table 1$)$.

In PVAC, 26 miRNAs were differentially expressed in tumours compared to normal tissues (Table 2). In details, 7 miRNAs showed higher expression levels in tumour than normal tissues, whereas 19 miRNAs were down-regulated. In this cohort, miR-323-3p showed the most significantly altered expression in tumour samples $(\mathrm{p}=0.0042)$, (Table 2$)$.

Table 3 reports differentially expressed miRNAs between tumour and normal tissues for both PDAC and PVAC. MiR-140-5p, miR-103, miR-1254, miR-199b-5p were significantly deregulated in both the subgroups of patients.

To ascertain the eventual existence of orthologous miRNAs, and to use them as internal methodological control, the human miRNAs that were found altered in PDAC and PVAC samples were checked in 71 other organisms probed in the microarray assay. All deregulated orthologous of human miRNAs showed a significant differential expression with similar fold-changes in these organisms, with the only exception of miR-182* and miR323-3p in the PDAC and PVAC subgroups of patients, respectively (Supplementary Table 1A-B).

\section{MiRNAs differentially expressed in PDAC versus PVAC}

In order to identify differentially expressed miRNAs between PDAC and PVAC patients, expression levels in paired tumour and normal tissues from PDAC and PVAC were compared. Twenty-seven miRNAs showed a significantly altered expression between PDAC and PVAC (Table 4). In details, 19 and 8 miRNAs were over- and down-regulated in PDAC compared to PVAC, respectively, with miR-889 and miR-323-3p showing the most relevant alteration (over- and down- expression, respectively) in PDAC vs PVAC.

Human miRNAs that were altered both in PDAC and PVAC were checked for the existence of orthologs in 71 organisms: all deregulated orthologs of human miRNAs showed a significant differential expression with close fold-changes, with the only exception of miR-323-3p (Supplementary Table 1C). 
Table 1: MicroRNA differentially expressed, by $t$-test, in paired normal and tumour tissues from patients with pancreatic ductal adenocarcinoma (PDAC)

\begin{tabular}{|c|c|c|c|c|c|}
\hline miRNA & Fold-change (T vs N) & P-value & miRNA & Fold-change (T vs N) & P-value \\
\hline hsa-miR-887 & 1.43 & $9.21 \mathrm{E}-05$ & hsa-miR-199b-5p & 1.57 & 0.0263 \\
\hline hsa-miR-125a-5p & 12.94 & 0.0021 & hsa-let-7d* & -1.70 & 0.0268 \\
\hline hsa-miR-1321 & -1.50 & 0.0024 & hsa-miR-503 & 1.80 & 0.0272 \\
\hline hsa-miR-744 & 2.25 & 0.0037 & hsa-miR-1244 & 2.46 & 0.0274 \\
\hline hsa-miR- $500^{*}$ & 3.30 & 0.0039 & hsa-miR-1254 & -1.59 & 0.0279 \\
\hline hsa-miR-526a & 1.34 & 0.0040 & hsa-miR-221 & 3.62 & 0.0283 \\
\hline hsa-miR-214 & 4.24 & 0.0040 & hsa-miR-27b* & 2.02 & 0.0285 \\
\hline hsa-miR-1267 & -2.26 & 0.0042 & hsa-miR-708 & 3.33 & 0.0292 \\
\hline hsa-miR-181a & 5.81 & 0.0043 & hsa-miR-132 & 2.80 & 0.0298 \\
\hline hsa-miR-1249 & -1.86 & 0.0048 & hsa-let-7i & 3.16 & 0.0306 \\
\hline hsa-miR-125a-3p & 1.96 & 0.0048 & hsa-miR-92b & 1.74 & 0.0306 \\
\hline hsa-let-7e & 12.59 & 0.0051 & hsa-miR-24 & 6.33 & 0.0310 \\
\hline hsa-miR-1237 & -1.68 & 0.0074 & hsa-miR-199a-5p & 4.90 & 0.0315 \\
\hline hsa-miR-181a* & -2.60 & 0.0081 & hsa-miR-29b & 1.36 & 0.0318 \\
\hline hsa-miR-134 & 1.84 & 0.0083 & hsa-miR-520a-3p & -1.40 & 0.0331 \\
\hline hsa-miR-29b-1* & 1.52 & 0.0083 & hsa-miR-378* & 1.66 & 0.0340 \\
\hline hsa-miR-132* & -2.47 & 0.0084 & hsa-miR-143 & 5.07 & 0.0340 \\
\hline hsa-miR-23a & 7.12 & 0.0087 & hsa-miR-16-2* & -1.46 & 0.0340 \\
\hline hsa-miR-559 & -2.59 & 0.0095 & hsa-miR-181 c* & -2.26 & 0.0341 \\
\hline hsa-miR-339-5p & 2.52 & 0.0098 & hsa-miR-30b & -1.92 & 0.0346 \\
\hline hsa-miR-154* & -1.87 & 0.0101 & hsa-miR-379 & 1.96 & 0.0352 \\
\hline hsa-miR-140-5p & 1.65 & 0.0101 & hsa-miR-136* & -1.43 & 0.0353 \\
\hline hsa-miR-181b & 4.82 & 0.0105 & hsa-miR-183* & -1.20 & 0.0373 \\
\hline hsa-miR-331-3p & 2.56 & 0.0114 & hsa-miR-100 & 2.43 & 0.0380 \\
\hline hsa-miR-92a & 4.00 & 0.0124 & hsa-miR-145 & 5.37 & 0.0380 \\
\hline hsa-let-7a & 8.04 & 0.0141 & hsa-miR-550 & 1.40 & 0.0392 \\
\hline hsa-miR-517* & -1.40 & 0.0153 & hsa-miR-26a & 5.03 & 0.0395 \\
\hline hsa-miR-30c & -1.81 & 0.0155 & hsa-miR-423-3p & 1.85 & 0.0405 \\
\hline hsa-miR-1301 & 2.14 & 0.0156 & hsa-miR-373 & -1.44 & 0.0412 \\
\hline hsa-miR-939 & 1.82 & 0.0159 & hsa-miR-107 & 5.30 & 0.0416 \\
\hline hsa-miR-1227 & -2.01 & 0.0164 & hsa-miR-219-1-3p & -1.66 & 0.0422 \\
\hline hsa-miR-487b & -2.12 & 0.0170 & hsa-miR-182* & 1.24 & 0.0423 \\
\hline hsa-miR-502-3p & 3.40 & 0.0171 & hsa-miR-103 & 2.71 & 0.0425 \\
\hline hsa-miR-125b & 4.06 & 0.0175 & hsa-miR-558 & -2.60 & 0.0427 \\
\hline hsa-miR-99b & 2.86 & 0.0176 & hsa-miR-23b & 6.00 & 0.0438 \\
\hline hsa-miR-32 & -1.20 & 0.0176 & hsa-miR-99a & 2.80 & 0.0440 \\
\hline
\end{tabular}

(Continued) 


\begin{tabular}{lccccc}
\hline miRNA & Fold-change (T vs N) & P-value & miRNA & Fold-change (T vs N) & P-value \\
\hline hsa-miR-199b-3p & 4.99 & 0.0178 & hsa-let-7g & 1.75 & 0.0444 \\
hsa-miR-374a & -2.39 & 0.0184 & hsa-miR-361-5p & 3.12 & 0.0445 \\
hsa-miR-21 & 4.33 & 0.0185 & hsa-miR-1246 & 2.28 & 0.0456 \\
hsa-let-7d & 4.73 & 0.0191 & hsa-miR-625* & -1.61 & 0.0457 \\
hsa-miR-1304 & -1.28 & 0.0196 & hsa-miR-130a & -1.53 & 0.0457 \\
hsa-miR-199a-3p & 5.55 & 0.0198 & hsa-miR-146a & 2.67 & 0.0459 \\
hsa-miR-498 & 1.51 & 0.0207 & hsa-miR-663b & 1.71 & 0.0459 \\
hsa-miR-324-3p & 1.72 & 0.0226 & hsa-miR-10a & 1.96 & 0.0464 \\
hsa-miR-99b* & 1.71 & 0.0229 & hsa-miR-431 & -1.42 & 0.0465 \\
hsa-miR-181d & 2.32 & 0.0238 & hsa-miR-27b & 2.62 & 0.0469 \\
hsa-miR-411 & -2.18 & 0.0242 & hsa-miR-1259 & -1.42 & 0.0476 \\
hsa-miR-34a & 2.81 & 0.0242 & hsa-miR-222 & 3.74 & 0.0485 \\
hsa-miR-195 & 3.24 & 0.0250 & hsa-miR-30d & -1.34 & 0.0487 \\
hsa-miR-196a & 1.34 & 0.0252 & hsa-miR-337-3p & -1.39 & 0.0494 \\
hsa-miR-382 & 2.37 & 0.0262 & & & \\
\hline
\end{tabular}

\section{Networks of correlated miRNAs in normal and tumour tissue samples}

\section{PDAC}

Networks of normal and tumour tissues were quite complex. The normal network was made of 98 miRNAs, wired by 1.232 correlation edges (Figure 1A). The diseased network was composed by 100 miRNAs connected by 1.434 links (Figure 1B). One thousand and one hundred and ninety and 542 out of the 1434 and 1232 links were preserved in the public dataset E-GEOD-60978. In details, 812 and 439 of these links had concordant correlation signs in PDAC and normal tissues, respectively, compared to our results (Supplementary Information \#Sheet A).

In PDAC, normal and tumour networks preserved several unchanged correlations for most of their miRNAs: only 4 were not shared by the two networks, including 1 (miR-348*) and 3 (miR-let-7d*, miR-1321, miR-373) out of the 98 and the 100 connected miRNAs in the normal and in the tumour networks, respectively. Conversely, 97 miRNAs were shared by the two networks including 65 linked by 775 edges, which represented more than $50-60 \%$ of the total number of the edges of both the networks, and 32 miRNAs which did not share any link between the normal and the tumour networks (Supplementary Table 2A).

Two (miR-1321 and miR-373-3p) out of the 3 miRNAs specifically belonging to the PDAC tumour network participate to the $A B C$ transporters signalling pathway (Supplementary Table 3). Among the miRNAs shared between normal and tumour networks
(Supplementary Table 2B), those that maintained the same links in the two networks $(\mathrm{n}=65)$ enriched 80 cancerrelated pathways, while 35 cancer-associated signalling events emerged for 7 out of the 32 miRNAs which did not share any links between normal and tumour networks. These miRNAs (miR-331-3p, miR-1246, miR-382, miR558, miR-181d, miR-1301, miR-559) exhibited high fold change values, and closely enriched 3 out of the 35 above mentioned signalling pathways.

As listed in Supplementary Table 2C, the most relevant signalling pathways for each subgroup of miRNAs were identified. In details, the pathways of the FOXO family and of MAPK were the most significantly impacted by the 65 shared miRNAs. The sets of 7 and 65 aforesaid miRNAs equally enriched the regulation of nuclear SMAD2/3 pathway, while ERBB1 and c-MET signalling pathways were enriched mainy by the 7 differentially connected miRNAs. The major pathways enriched by the 7 differentially connected miRNAs were the ERBB1 and c-MET signalling pathways. In addition, the Notch mediated HES/HEY network resulted to be enriched solely by the subgroup of the 7 miRNAs.

\section{PVAC}

Both normal-adjacent and tumour PVAC networks were much smaller than the corresponding PDAC networks. In particular, the normal network was made of 17 nodes and 15 edges. Topologically, this network was characterized by a long chain of expression correlations among 7 miRNAs (miR-103, miR-889, miR-29b-2*, miR-410, miR-30b*, miR-1280 and miR-1228*), by 2 closed groups (triangles) including miR-548b-3p, miR-1254, miR-490-5p (triangle 
Table 2: MicroRNA differentially expressed, by $t$-test, in paired normal and tumour tissues from patients with adenocarcinoma of papilla of Vater (PVAC)

\begin{tabular}{|c|c|c|}
\hline miRNA & Fold-change (T vs N) & P-value \\
\hline hsa-miR-323-3p & 1.59 & 0.0042 \\
\hline hsa-miR-525-5p & -1.22 & 0.0111 \\
\hline hsa-miR-199b-5p & -1.25 & 0.0120 \\
\hline hsa-miR-30d ${ }^{*}$ & -1.27 & 0.0130 \\
\hline hsa-miR-339-3p & -3.35 & 0.0202 \\
\hline hsa-miR-490-5p & -1.34 & 0.0208 \\
\hline hsa-miR-1305 & -1.69 & 0.0232 \\
\hline hsa-miR-1270 & -1.35 & 0.0273 \\
\hline hsa-miR-1254 & -2.22 & 0.0292 \\
\hline hsa-miR-643 & 1.55 & 0.0303 \\
\hline hsa-miR-29b-2* & 1.73 & 0.0309 \\
\hline hsa-miR-509-5p & -1.10 & 0.0316 \\
\hline hsa-miR-200c ${ }^{*}$ & 1.38 & 0.0335 \\
\hline hsa-miR-410 & -1.51 & 0.0337 \\
\hline hsa-miR-563 & -1.30 & 0.0343 \\
\hline hsa-miR-187* & -1.49 & 0.0345 \\
\hline hsa-miR-889 & -6.53 & 0.0352 \\
\hline hsa-miR-551a & -1.22 & 0.0394 \\
\hline hsa-miR-30b* & -1.58 & 0.0397 \\
\hline hsa-miR-140-5p & 1.43 & 0.0399 \\
\hline hsa-miR-1280 & -3.97 & 0.0401 \\
\hline hsa-miR-548b-3p & 1.29 & 0.0423 \\
\hline hsa-miR-1228* & -4.07 & 0.0432 \\
\hline hsa-miR-551b* & -1.17 & 0.0442 \\
\hline hsa-miR-5481 & -1.37 & 0.0479 \\
\hline hsa-miR-103 & 15.43 & 0.0485 \\
\hline
\end{tabular}

1) and miR-339-3p, miR-509-5p, miR-140-5p (triangle 2), respectively, and by two pairs of miRNAs (miR-551b*/miR$30 \mathrm{~d}^{*}$ and $\left.\mathrm{miR}-551 \mathrm{a} / \mathrm{miR}-187^{*}\right)$, (Figure $2 \mathrm{~A}$ ).

The tumour network was made of 21 miRNAs linked by 17 edges. No closed groups resulted, whereas it included a long chain of 8 miRNAs (miR-1280, miR-1228*, miR323-3p, miR-551a, miR-490-5p, miR-103, miR-1270 and miR-29b-2*), three shorter chains of 5 miRNAs (miR1254, miR-889, miR200c*, miR-410 and miR-5481) and 3 miRNAs (miR-563/miR-30b ${ }^{*} / \mathrm{miR}-140-5 \mathrm{p}$ and $\mathrm{miR}-551 \mathrm{~b}^{*} /$ miR-509-5p/miR-199b-5p), and one pair of miRNAs (miR-548b-3p/miR-30d*). In particular, the longest chain was made of an odd number (five) of consecutive inverse correlations (miR-1270, miR-103, miR-490-5p, miR-551a,
miR-323-3p, miR-1228*), of which miR-103 was the only miRNA to be highly expressed (Figure 2B).

Comparing normal with tumour networks of PVAC samples, we found 15 miRNAs and only 1 edge in common, whereas 6 miRNAs (miR-1270, miR323-3p, miR-200c*, miR-548I, miR-563, miR-199b$5 \mathrm{p})$ were included only in the diseased network. By pathway enrichment analysis, 3 out of these 6 miRNAs were involved in the LICAM interactions process [miR-199b-5p (AKN2, CLTC, LAMC1), miR-323a-3p (ANK2, DCX, KCNQ3, KIAA1598, LAMC1, NRP2, SCN2A, SPTBN1), miR-563 (ITGA9, SPTBN2)], and in the axon guidance signalling pathway [miR-199b-5p (ANK2, ARHGEF12, CLTC, GSK3B, LAMC1, MYH9, 
Table 3: MicroRNA showing significant or a trend of alteration in tumour compared to normal tissues samples in both pancreatic ductal adenocarcinoma (PDAC) and adenocarcinoma of papilla of Vater (PVAC)

\begin{tabular}{|c|c|c|c|c|}
\hline & \multicolumn{2}{|c|}{ PDAC } & \multicolumn{2}{|c|}{ PVAC } \\
\hline & Fold-change ( $\mathrm{T}$ vs $\mathrm{N}$ ) & P-value & Fold-change (Tvs N) & P-value \\
\hline hsa-miR-140-5p & 1.65 & 0.0101 & 1.43 & 0.0399 \\
\hline hsa-miR-103 & 2.71 & 0.0425 & 15.43 & 0.0485 \\
\hline hsa-miR-1254 & -1.59 & 0.0279 & -2.22 & 0.0292 \\
\hline hsa-miR-199b-5p & 1.57 & 0.0263 & -1.25 & 0.0120 \\
\hline hsa-miR-1244 & 2.46 & 0.0274 & 7.48 & 0.0579 \\
\hline hsa-miR-146a & 2.67 & 0.0459 & 5.02 & 0.0593 \\
\hline hsa-miR-30d & -1.34 & 0.0487 & -4.56 & 0.0556 \\
\hline hsa-miR-1259 & -1.42 & 0.0476 & 1.25 & 0.0606 \\
\hline hsa-miR-1304 & -1.28 & 0.0196 & 1.15 & 0.0828 \\
\hline hsa-miR-525-5p & -1.18 & 0.0837 & -1.22 & 0.0111 \\
\hline hsa-miR-643 & -1.13 & 0.0903 & 1.55 & 0.0303 \\
\hline hsa-miR-1207-5p & 2.08 & 0.0722 & 2.08 & 0.0771 \\
\hline hsa-miR-138-1* & 1.54 & 0.0807 & 2.10 & 0.0526 \\
\hline hsa-let-7f-1 ${ }^{*}$ & -1.58 & 0.0589 & -1.51 & 0.0623 \\
\hline hsa-miR-629* & -2.25 & 0.0716 & -5.11 & 0.0722 \\
\hline hsa-miR-1260 & -1.63 & 0.0809 & -2.02 & 0.0982 \\
\hline hsa-miR-302a* & -1.64 & 0.0808 & 1.24 & 0.0546 \\
\hline
\end{tabular}

PLXNA2, RGMA, RGMB, RND1, SEMA6A, SRGAP2), miR-323a-3p (ANK2, CLASP1, CREB1, DCX, ENAH, KCNQ3, KIAA1598, KRAS, LAMC1, MET, NRP2, PAK7, PITPNA, ROCK1, SCN2A, SEMA6D, SPTBN1, SRGAP1), miR-563 (COL1A2, COL3A1, ITGA9, SPTBN2)]. Conversely, miR-1270, miR-200c ${ }^{*}$, miR-548I did not enrich any pathway related to pancreatic cancer.

Focusing on miRNAs with elevate fold-change values in the two networks, namely on miR-1254 (FC=$2.22)$, miR-1228* $(\mathrm{FC}=-4), \mathrm{miR}-1280(\mathrm{FC}=-3.97)$, miR$889(\mathrm{FC}=-6.53)$ and miR-103 $(\mathrm{FC}=15.4)$, we noticed that the only common correlation was that between miR$1228^{*}$ and miR-1280. MiR-889 was directly linked to and inversely correlated with miR-103 in normal tissues, while it was directly correlated with miR-1254 in tumour tissues. However, only miR-103 was directly involved in the regulation of pancreatic cancer-associated processes: E-cadherin signalling events (CDH1), TGF beta signalling pathway (TGFBR2, APC, CDH1, CREBBP, EP300, SMAD3, TSC2), and altered TFG-beta SMAD dependent signalling (TGFBR2, SMAD3, and FBXW7).

Finally, the two triangles of miRNAs were peculiar to the normal network only, and among their validated target genes retrieved by miRTarBase, miRWalk and TarBase 7 there were DICER1 (controlled by miR- 548b-3p), TP53 and CDH1 (regulated by miR-140-5p, belonging to the triangle 2). These genes are mostly involved in the perturbation of DICER1, p53, p21, and E-cadherin pathways.

\section{Networks of correlated miRNAs in tumour versus normal tissue samples}

\section{PDAC}

A correlation-based network was built with the differentially expressed miRNAs between healthy-adjacent and tumour tissues, and drawn in Figure 3. This was clustered in 5 highly cohesive subgroups of miRNAs: cluster 1 with 38 miRNAs (density: 0.504, p-value: 1.405E-12); cluster 2 with 18 miRNAs (density: 0.608, p-value: $2.556 \mathrm{E}-5$ ); cluster 3 with 5 miRNAs (density: 0.700, p-value: 0.007); cluster 4 with 4 miRNAs (density: 0.833, p-value: 0.025 ), and cluster 5 with 8 miRNAs (density: 0.571, p-value: 0.077). The complete lists of miRNAs belonging to each cluster is reported in Supplementary Table 4.

A ranking of these miRNAs was assessed by the calculation of an array of topological indices (cf. Methods). We verified that 9 miRNAs (miR-34a, miR125a-5p, miR-199a-5p, miR-181a, miR-30c, miR-30b, miR-339-5p, miR-214, miR-411) belonging to cluster 1 
Table 4: MicroRNA differentially expressed in paired normal and tumour tissues, by $t$-test, in pancreatic ductal adenocarcinoma (PDAC) compared to adenocarcinoma of papilla of Vater (PVAC)

\begin{tabular}{|c|c|c|}
\hline miRNA & Fold change (PDAC vs PVAC) & P-value \\
\hline hsa-miR-323-3p & -2.13 & 0.0006 \\
\hline hsa-miR-199b-5p & 2.04 & 0.0024 \\
\hline hsa-miR-1259 & -1.93 & 0.0024 \\
\hline hsa-miR-1304 & -1.50 & 0.0067 \\
\hline hsa-miR-550 & 2.30 & 0.0130 \\
\hline hsa-miR-122 & -1.55 & 0.0145 \\
\hline hsa-miR-302a* & -2.08 & 0.0157 \\
\hline hsa-miR-922 & -1.99 & 0.0183 \\
\hline hsa-miR-125a-3p & 2.33 & 0.0195 \\
\hline hsa-miR-643 & -1.83 & 0.0236 \\
\hline hsa-miR-1301 & 2.50 & 0.0249 \\
\hline hsa-miR-518c* & 1.41 & 0.0259 \\
\hline hsa-miR-889 & 7.42 & 0.0317 \\
\hline hsa-miR-516a-3p & 1.66 & 0.0332 \\
\hline hsa-miR-939 & 1.69 & 0.0335 \\
\hline hsa-miR-125b-1* & 1.99 & 0.0336 \\
\hline hsa-miR-187* & 1.73 & 0.0339 \\
\hline hsa-miR-18b* & 3.09 & 0.0349 \\
\hline hsa-miR-548b-3p & -2.07 & 0.0369 \\
\hline hsa-miR-1270 & 1.54 & 0.0369 \\
\hline hsa-miR-593 & 1.31 & 0.0382 \\
\hline hsa-miR-410 & 1.64 & 0.0415 \\
\hline hsa-miR-551b* & 1.58 & 0.0435 \\
\hline hsa-miR-23b* & 1.60 & 0.0452 \\
\hline hsa-miR-490-5p & 1.86 & 0.0456 \\
\hline hsa-miR-339-3p & 2.78 & 0.0457 \\
\hline hsa-miR-450a & 1.58 & 0.0496 \\
\hline
\end{tabular}

were top-five ranked for at least one topological index, and that 3 of these (miR-181a, miR-30c, miR-30b) exhibited the highest values for more than one topological index (Supplementary Table 5A).

The genes targeted by miRNAs belonging to cluster 1 were mostly controlled by 25 out of 38 miRNAs, and a few of these targeted the most genes (Supplementary Table 5B). By pathway enrichment analysis, the altered $T G F$ beta signalling pathway was regulated by TGFBR2 (miR21, miR-214), SMAD3 (miR-21, miR-146a, miR-30b, miR-195, miR-503), and SMAD4 (miR-21, miR-146a, miR-181a, miR-181a*, miR-181b, miR-181c*, miR-181d) (Supplementary Table 5C).

\section{PVAC}

The network wiring the 26 deregulated miRNAs of PVAC tumours was drawn in Figure 4. The clustering algorithm identified only one cluster, which included miR-1254, miR-200c*, miR-1270, miR-889, miR551b*, miR-103, miR-29b-2* and miR-548I (density: 0.533, p-value: 0.004) and so MiR-548I, miR-103 and miR-1254 worked as "seeding nodes" of the clustering method, and miR-29b-2-5p was directly correlated with two of the three seeding nodes (miR-103 and miR-1254). Additionally, 10 miRNAs were correlated in pairs: miR551a/miR563, miR-410/miR30b*， miR-548b-3p/miR- 
323-3p, miR-1305/miR-509-5p, miR-30d*/miR-1280. The remaining $8 \mathrm{miRNAs}$ did not show any significant correlation with any other miRNAs.

We found that 3 out of the 8 miRNAs (miR-103, miR29b-2* miR-200c*) controlled several genes (Supplementary Table 6A), which turned out to enrich different signalling pathways: TGF beta pathway (TGFBR2, APC, CDH1, CREBBP, EP300, SMAD3, TSC2), altered TFG-beta SMAD dependent signalling (TGFBR2, SMAD3, FBXW7), p53 pathway and transcriptional activation of cell cycle inhibitor p21 (TP53), and E-cadherin signalling events (CDH1), (Supplementary Table 6B).

\section{Networks matching}

Four miRNAs were significantly deregulated both in PDAC and in PVAC. The regulation directions of miR-103 and miR-1254 were concordant, yet with different foldchanges values: miR-103 (PDAC: $\mathrm{FC}=2.71$; PVAC: $\mathrm{FC}$
$=15.43$ ) and miR-1254 (PDAC: FC = -1.59; PVAC: FC $=2.22$ ). MiR-140-5p and miR-199b-5p were mildly altered in both the tumour entities, with opposite directions of regulation for the latter miRNA [miR-140-5p: $\mathrm{FC}=1.65$ (PDAC), $F C=1.43$ (PVAC); miR-199b-5p: $\mathrm{FC}=1.57$ $(\mathrm{PDAC}) ; \mathrm{FC}=-1.24(\mathrm{PVAC})]$.

\section{miR-103}

In PDAC, miR-103 was significantly correlated with 17 miRNAs that were not regulated less than 2 folds in tumour compared to normal tissues. Almost all correlations were positive, except from those involving miR-1227, miR1267 and miR-558 (Figure 5). It is worth noticing that 15 on 17 miRNAs fell in the cluster 2 (Supplementary Table 4). Topologically, miR-92a was in the top-ten ranked for betweenness (0.0499), while let-7a, let-7d and miR-145 were the most important in terms of correlation coefficient values (0.857, 0.727 and 0.705, respectively). MiR-103 targeted directly FBXW7, VCP, CHD1, PCDH17, HIP1, TGFBR2,

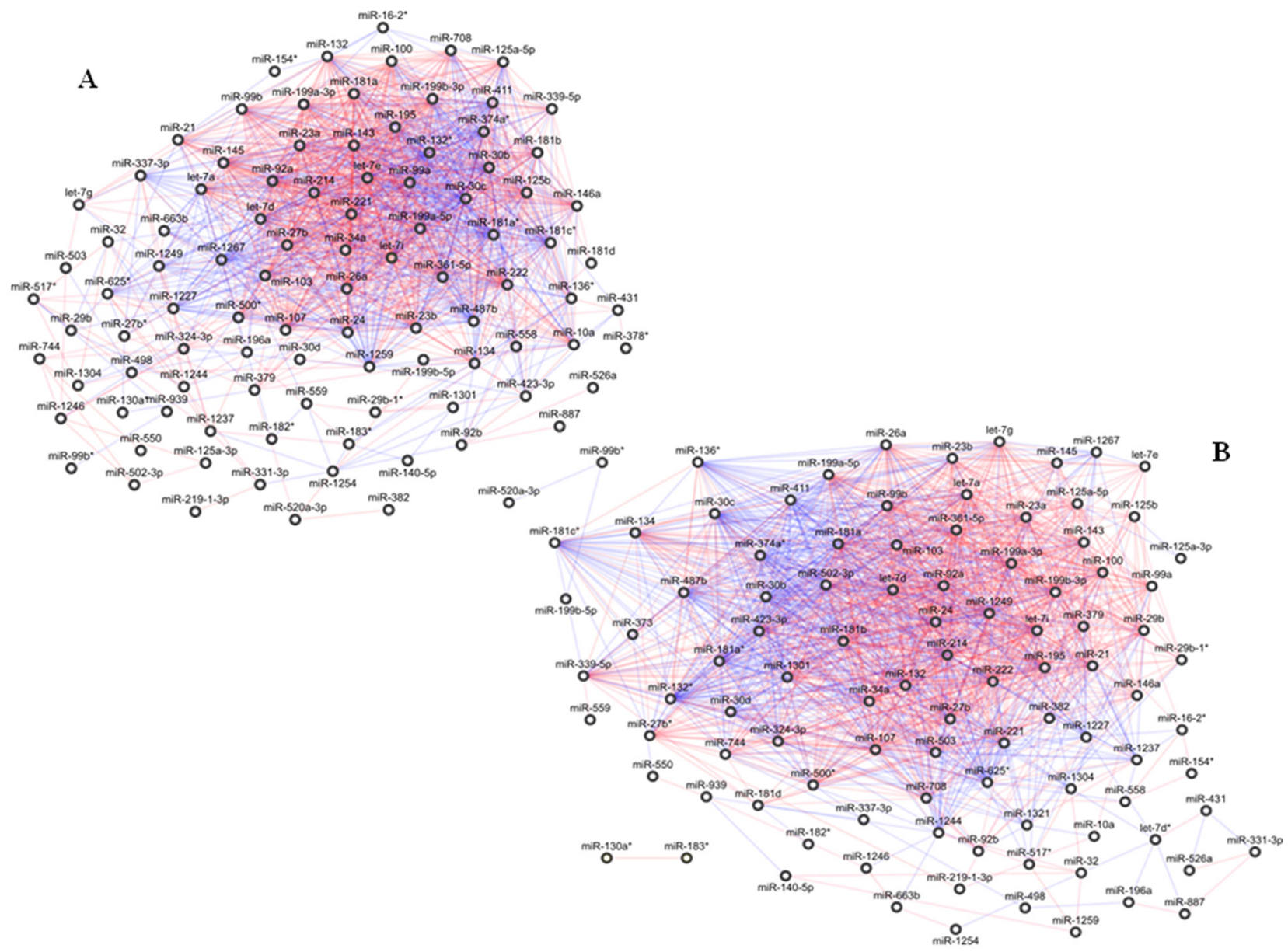

Figure 1: Correlation network between microRNA altered in tissue samples from patients with pancreatic ductal adenocarcinoma (PDAC). (A) Normal tissues; global geometric attributes: connected components 1, network diameter 6, network centralization 0.27 , shortest paths $9506(100 \%)$, characteristic path length 2.35 , average number of neighbours 25.14 , network density 0.26 , and network heterogeneity 0.77. (B) Tumour tissues; global geometric attributes: connected components 3 , network diameter 6, network centralization 0.32 , shortest paths $89.38(90 \%)$, characteristic path length 2.18 , average number of neighbours 28.68 , network density 0.29 , and network heterogeneity 0.72 . Direct and inverse correlations are in red and blue, respectively. 
TNF genes, while indirectly, through 11 of its 17 neighbour miRNAs, several more genes (Supplementary Table 7A). The majority of these genes were controlled by let-7d-5p, let-7a5p, let-7e and miR-92a, and CDKN2A, FBXW7, TP53 and MYC were targeted by the most miRNAs. Some of the target genes, i.e., TGFBR2, SMAD3, SMAD4 regulated by (miR103, miR-92a, miR-26a-5p, miR-145-5p, miR-143, let-7d-5p, miR-23a-3p, miR-107) turned out to participate to the $T G F$ beta signalling pathway in PDAC (Supplementary Table 7B).

In PVAC, miR-103 was positively correlated only with miR-551b*, which was in turn positively correlated with miR-29b-2* and negatively with miR-889 (Figure 4). About predicted targets, the seven genes mentioned above were regulated by miR-103, whereas 23 genes were targeted by miR-29b-2* (Supplementary Table 6A). These genes over-represented a number of signalling pathways: TGF beta pathway (TGFBR2, APC, CDH1, CREBBP, EP300, SMAD3, TSC2), altered TFG-beta SMAD dependent signalling (TGFBR2, SMAD3, FBXW7), p53 pathway and transcriptional activation of cell cycle inhibitor p21 (TP53), and E-cadherin signalling events (CDH1), (Supplementary Table 6B).

\section{miR-1254}

In PDAC, miR-1254 was directly correlated only with let-7g, which in turn was correlated with miR-221 and miR-92b. MiR-1254 did not target directly any known genes associated with the disease, and no target genes were found to be regulated by miR-92b. Conversely, let-7g was confirmed to target 9 critical genes (TP53, CDKN2A, FBXW7, FN1, MYC, TBX5, GLI1, TNF, ERCC4), and miR-221 resulted to control only 4 of these (TP53, CDKN2A, MYC, TNF).

In PVAC, miR-1254 was negatively correlated only with miR-200c*, which in turn was directly correlated with miR-29b-2* and miR-1270 (Figure 5). As for miR-1254, no target genes of miR-1270 were reported to be directly associated to the disease. Conversely, miR-200c ${ }^{*}$ and 29b-2* targeted several genes listed in Supplementary Table 5A.

Among the enriched signalling pathways, it emerges that the DNA damage repair pathway was shared by PDAC and PVAC. In details, the neighbourhood of miR1254 was more involved in the regulation of this pathway: both miR-200c* (correlated to miR-1254 in PVAC) and
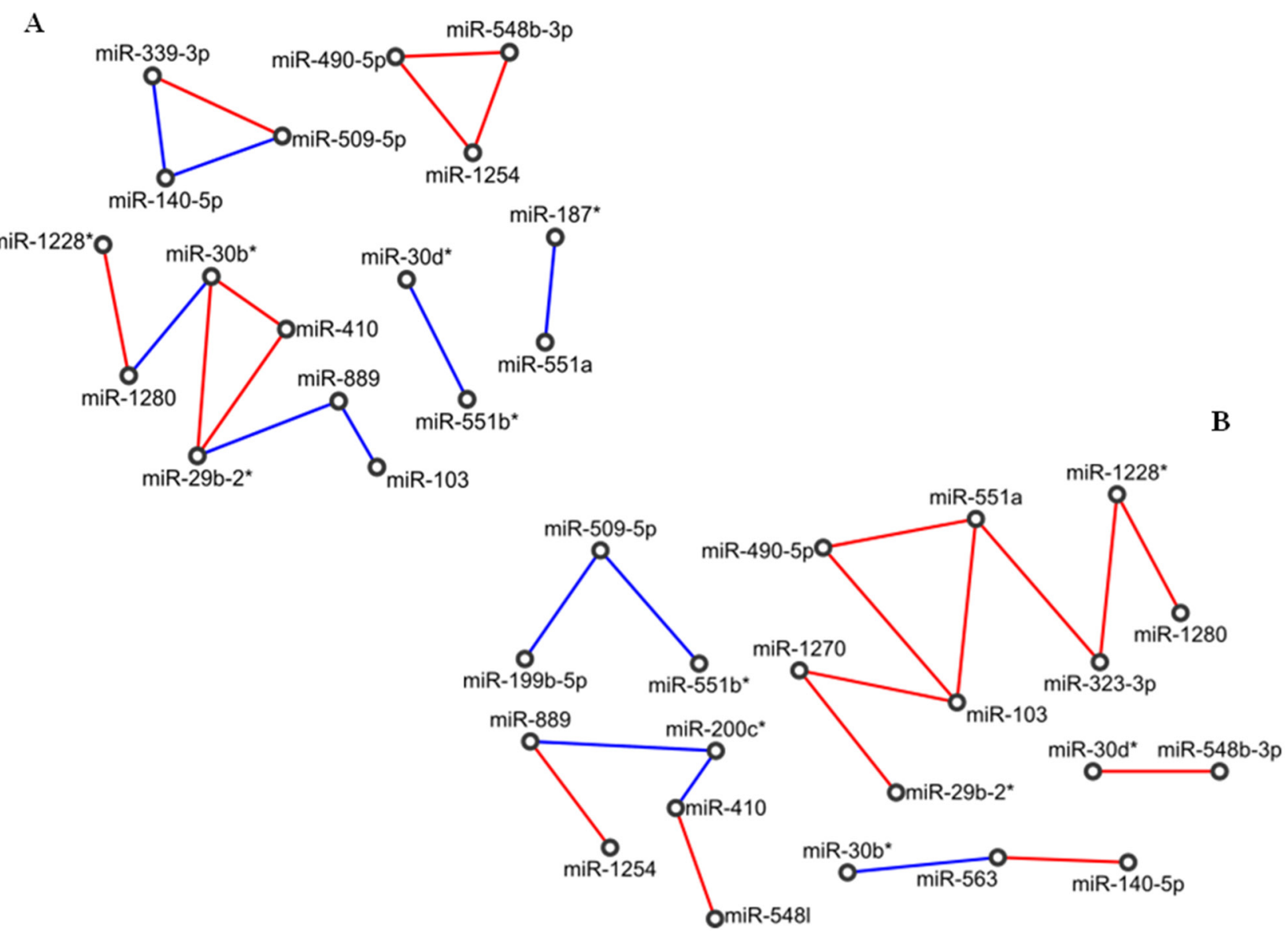

Figure 2: Correlation network between microRNA altered in tissue samples from patients with adenocarcinoma of papilla of Vater (PVAC). (A) Normal tissues; (B) tumour tissues. Direct and inverse correlations are in red and blue, respectively. 
let-7g (correlated to miR-1254 in PDAC) targeted EP300 and ERCC4 genes, respectively.

\section{miR-140-5p}

In PDAC, miR-140-5p was correlated with 4 miRNAs (miR-939, miR-625*, miR-1249 and miR-27b$3 p)$. Genes associated with the pancreatic cancer core signalling pathway were controlled only two of these
miRNAs, namely miR-140-5p and miR-27b-3p. As shown in Supplementary Table 8A, these two miRNAs were predicted to regulate the same cancer-related target genes. By pathway enrichment analysis, several signalling pathways were uncovered: $p 53$ pathway, the transcriptional activation of cell cycle inhibitor p21 (TP53), E-cadherin signalling events (CDH1), (Supplementary Table 8B).

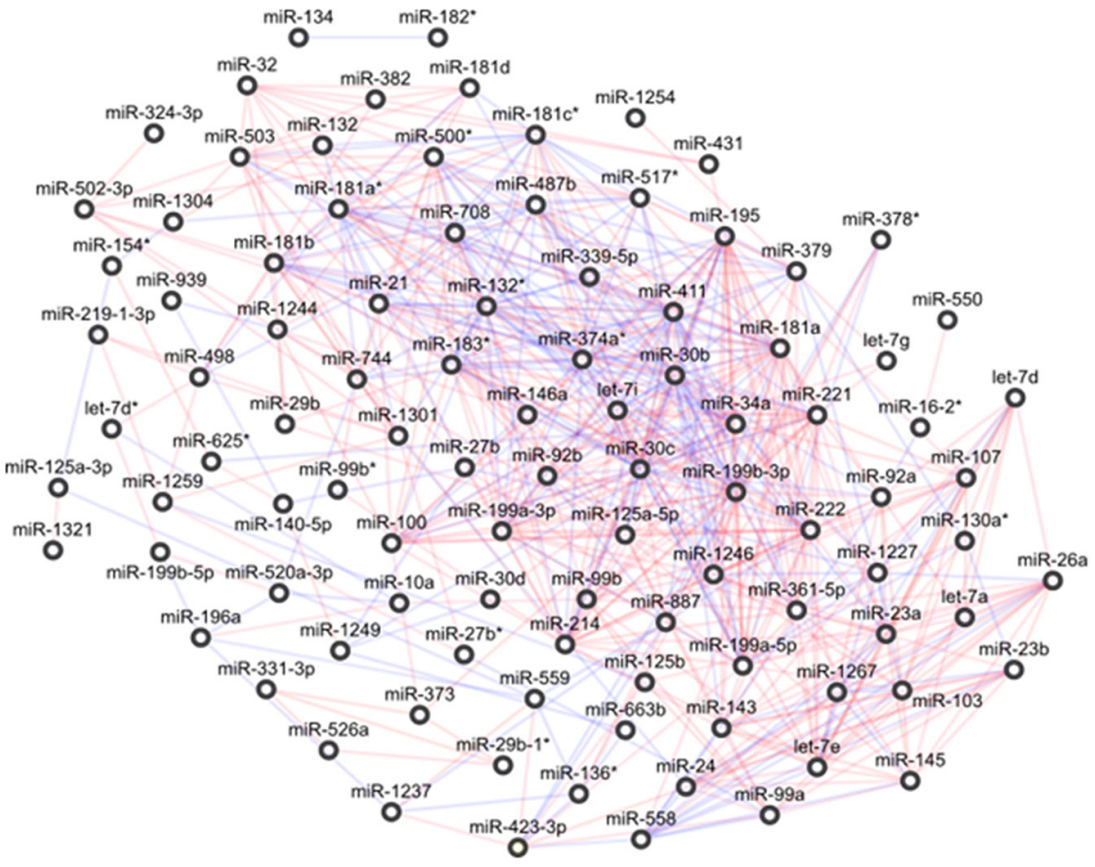

\begin{tabular}{|lc|}
\hline Measure & Value \\
Clustering coefficient & 0.481 \\
Conneded components & 2 \\
Diameter & 8 \\
Radius & 1 \\
Centralization & 0.221 \\
Shortest path & $9508(96 \%)$ \\
Characteristicpathlength & 2.746 \\
Avg. number ofneighbors & 12.54 \\
Number ofnodes & 100 \\
Density & 0.127 \\
Heterogeneity & 0.750 \\
Isolated nodes & 0 \\
Number of self-loops & 0 \\
Multi-edge node pairs & 0 \\
\hline
\end{tabular}

Figure 3: Correlation network between microRNA altered in tumour compared to normal tissue samples from patients with pancreatic ductal adenocarcinoma (PDAC). Direct and inverse correlations are in red and blue, respectively.

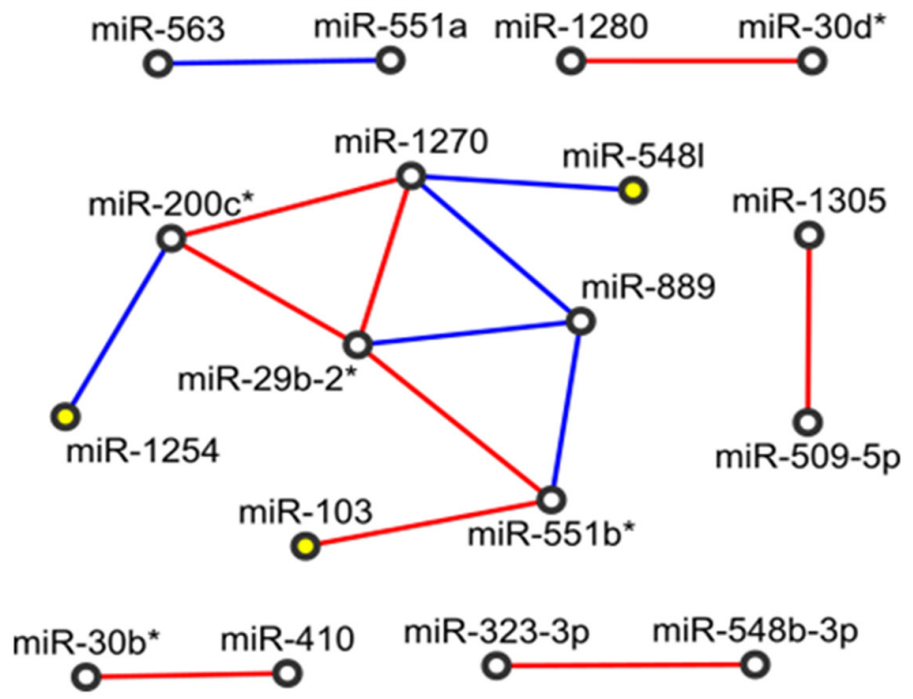

\begin{tabular}{|lc|}
\hline Measure & Value \\
Clustering coefficient & 0.120 \\
Conneded components & 6 \\
Diameter & 4 \\
Radius & 1 \\
Centralization & 0.154 \\
Shortest path & $66(21 \%)$ \\
Characteristicpathlength & 1.848 \\
Avg.number ofneighbors & 1.667 \\
Number ofnodes & 18 \\
Density & 0.098 \\
Heterogeneity & 0.663 \\
Isolated nodes & 0 \\
Number ofself-loops & 0 \\
Multi-edge node pairs & 0 \\
\hline
\end{tabular}

Figure 4: Correlation network between microRNA altered in tumour compared to normal tissue samples from patients with adenocarcinoma of papilla of Vater (PVAC). Direct and inverse correlations are in red and blue, respectively, and seeding nodes are colored in yellow. 
Conversely, miR-140-5p did not correlate with any miRNA significantly deregulated in tumours, compared to normal tissues in PVAC patients. Therefore, the regulation of the above-mentioned signalling pathways might be imputed only to miR-140-5p in PVAC.

\section{miR-199b-5p}

In PDAC, miR-199b-5p was characterized by relevant topological values: average shortest path (3.8865) and topological coefficient (0.5238). Its expression was correlated with 3 miRNAs: directly with both miR-520a-3p and let-7d*, and inversely, with miR-196a. MiR-199b-5p did not directly target any known PDAC gene. However, part of its closest neighbourhood regulated several genes (Supplementary Table 9A). By pathway enrichment analysis, several signalling pathways were uncovered: $p 53$ pathway, the transcriptional activation of cell cycle inhibitor p21 (TP53), E-cadherin signalling events (CDH1), (Supplementary Table 9B).

Conversely, miR-199b-5p did not correlate with any miRNAs in the PVAC network.

\section{MiRNAs differentially expressed in PDAC versus PVAC}

MiR-889 (FC=7.4) and miR-323-3p $(\mathrm{FC}=-2.12)$ were the most discriminating miRNAs between PDAC and PVAC. No genes associated with pathways involved in PDAC/PVAC were found to be targeted by miR-889, whereas for miR-323-3p two validated targets genes were identified (NTRK1 and TNF), which were involved in the biological process of pain perception $(\mathrm{p}=1.421 \mathrm{E}-2)$.

The 27 miRNAs that resulted significantly altered between the matched-pairs of tissues of PDAC and PVAC significantly influenced the Wnt and Hedgehog signalling pathways, other than $M A P K, E r b B 1$ and Notch (already associated to PDAC) and TFG-Beta and p53 (already associated to PVAC) signalling pathways.

\section{DISCUSSION}

In the last years, several studies have investigated miRNA expression alterations in pancreatic cancer. However, the most were focused on PDAC while only a few investigated the expression profiles of miRNA in other pancreatic diseases, including PVAC [15-18].

Our study was designed to dig miRNA expression alterations out of PDAC or PVAC, and to figure out if these could be associated with distinctive signalling pathways in turn reflecting differences in pathogenesis and clinical behaviours of these two different entities of cancer.

Overall, PDAC and PVAC showed different miRNA alterations, with PDAC being more deregulated and probably a more complex disease. Changes in miRNA expression levels displayed a different direction in the two subgroups of patients. In PDAC samples, 68 out of the 101 altered miRNAs $(67 \%)$ were overexpressed in tumours compared to normal tissues. These miRNAs may function as oncogenes promoting cancer development by negatively regulating tumour suppressor genes, whose aberrations were known to synergistically accelerate the progression of pancreatic carcinogenesis through pre-neoplastic lesions to adenocarcinoma [19, 20]. Conversely, more miRNAs were down-regulated in tumours compared to normal tissue samples from PVAC patients $(19 / 26,73 \%$ vs $7 / 26,27 \%)$; the high percentage

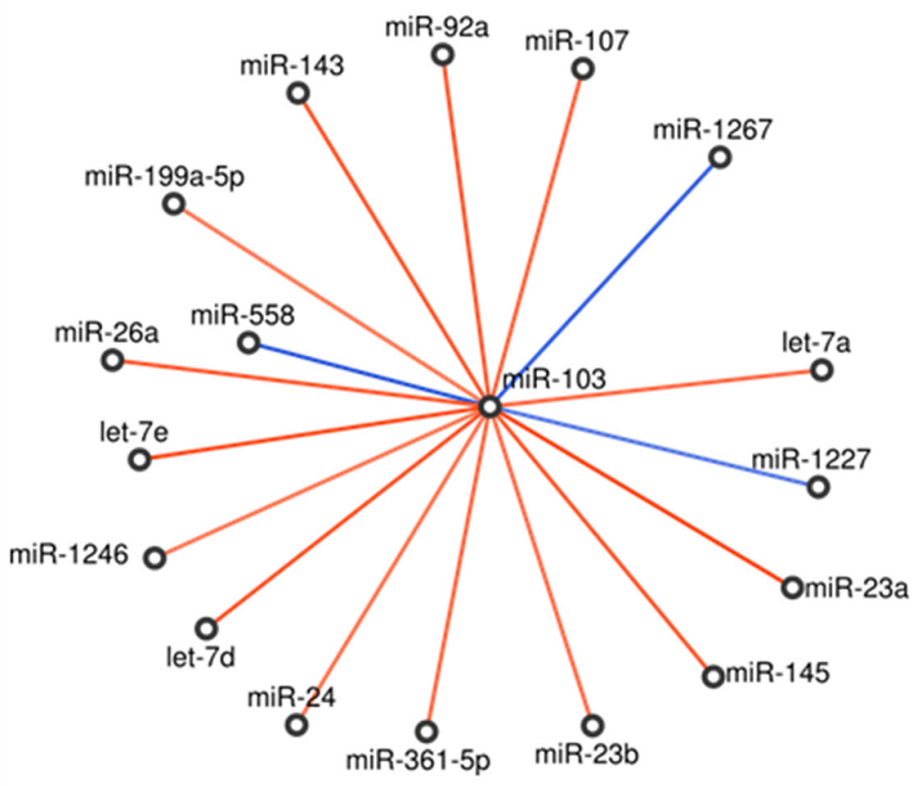

\begin{tabular}{|lc|}
\hline Measure & Value \\
Average shortest Path Length & 2.546 \\
Betweenness & 0.0059 \\
Closeness & 0.393 \\
Clustering coefficient & 0.596 \\
Degree & 17 \\
Number ofneighbors & 17 \\
Eccentricity & 5 \\
Neighborhood connectivity & 16.294 \\
Radiality & 0.807 \\
Stress & 928.0 \\
Topological coefficient & 0.362 \\
\hline
\end{tabular}

Figure 5: MiR-103 neighbourhoods in pancreatic ductal adenocarcinoma (PDAC). Direct and inverse correlations are in red and blue, respectively. 
of under-expressed miRNAs in PVAC may function as tumour suppressor genes and may inhibit cancer by positively regulating oncogenes. With respect of the molecular changes, ampullary cancer was seen to be more similar to the intestinal cancer and characterized by alterations in oncogenes rather than in tumour suppressor genes $[21,22]$.

By comparing miRNA expression alterations in PDAC and PVAC we found that only 4 miRNAs (miR-140-5p, miR-103, miR-1254, miR-199b-5p) were deregulated in both the subgroups of patients. Conversely, a limited overlap between miRNAs significantly altered in both PDAC and PVAC emerged, suggesting the existence of tumour-specificity for miRNAs alteration in different entities of pancreatic cancer.

Furthermore, the expression of 27 miRNAs was significantly different in PDAC compared to PVAC. One of these, miR-323-3p, was shown to influence the biological process of pain perception. Pain has long been considered the most common symptom in patients with pancreatic disorders, and its presence in newly diagnosed patients with potentially operable pancreatic cancer is a predictor of resectability and survival [23]. However, the pattern of pain sensation process does not characterized different pancreatic tumours equally, while it was reported to depend on tumour type, anatomic localization and dignity of different pancreatic diseases [24]. This miRNA was already reported in literature for its association with painful events, such as ectopic pregnancy. Authors found that miR-323$3 p$ was significantly increased in women experiencing abdominal pain/cramping and that received a diagnosis of ectopic pregnancy compared to those with intrauterine pregnancy and spontaneous abortion [25]. In our series miR-323-3p was altered in the opposite direction, so we can hypothesize that it can influence the pattern of pain sensation in PDAC and PVAC which clinically distinguishes these two tumour entities.

In order to identify the most relevant miRNAs in each tumour entity, and the signalling pathways in which there are involved, expression data were used to wire correlation networks both for PDAC and PVAC. MiRNA interactions in normal and tumour tissues were first taken into account in order to highlight the main alterations characterizing the two tissue types. Afterwards, with the intent to uncover those alterations that further exalt the differences between normal and tumour, the correlation networks were built by using miRNAs differentially expressed in matched pairs of tissues. By this approach, it was possible to identify three main subgroups of miRNAs either in PDAC and PVAC: miRNAs present only in the diseased network, those shared between normal and tumour network, and finally the most relevant miRNAs significantly altered in tumour compared to normal tissues.
In PDAC the diseased network was denser, more reachable and complex compared to the normal one. Indeed, it exhibited a shorter diameter, a higher centralization and a greater number of node neighbours, even though its local betweenness centrality was, in the average, lower than that of the nodes of the normal network. In general, the increased complexity of tumour compared to normal network might reflect the substantial reorganization of the controlling mechanisms fulfilled by these miRNAs in cancer. By comparing normal and tumour networks, we found that only a few miRNAs were not shared between the two networks, including 3 out of the 100 miRNAs connected in the tumour network (let-7d*, miR-1321, miR-373). By pathway enrichment analysis, $\mathrm{ABC}$ transporters signalling pathway emerged as regulated by miR-1321 and miR-373-3p.

Drug resistance is a major obstacle to the successful chemotherapy, and the ATP-binding cassette (ABC) transporter family members are the most common genes involved in the cancer multidrug resistance $[26,27]$. We found that eight $\mathrm{ABC}$ proteins were targeted by the miR-1321 and miR-373-3p in PDAC network. Three out of them (ABCC2, ABCC5, ABCC 8 ) have been already described for their association with drug resistance in pancreatic cancer [28-33]. Furthermore, a possible involvement of $\mathrm{ABCC} 8$ in pancreatic adenocarcinoma development and progression was recently reported [29].

By comparing normal and tumour networks in PDAC, we identified 97 miRNAs in common between the two networks including a core set of miRNAs that is partly or completely modulated by the disease ( 65 miRNAs which preserved unchanged correlations), and another set of 32 miRNAs which do not share any links between normal and tumour networks. In the latter subgroup we also identified 7 miRNAs characterized by high foldchange values. Among the most relevant pathways regulated by these subsets of miRNAs, we hypothesized that the Notch-mediated HES/HEY network, enriched only by the 7 miRNAs not sharing links, might be involved in the tumor progression compared to the pathways enriched by miRNAs belonging also to the subset of 65 miRNAs, which instead might be involved in the onset of the disease. In addition, according to the score values associated to each pathway, it was possible to suppose a sequential involvement for these signalling events in the carcinogenesis of pancreatic cancer: FOXO family and MAPK signalling pathways might intervene before, followed by regulation of nuclear SMAD2/3 signalling, while molecular events mediated by ERBB1 and c-MET might be deregulated later during the development of pancreatic cancer.

The importance of all these signalling pathways was supported by the literature. Most studies have revealed that the Notch activation has an oncogenic role for pancreatic cancer and is involved in cell proliferation, apoptosis, migration, invasion, metastases, and angiogenesis [34-41]. On the other hand, the mammalian forkhead members of 
the class $\mathrm{O}(\mathrm{FOXO})$ transcription factors are implicated as tumour suppressors in the regulation of several biological processes, including stress resistance, metabolism, cell cycle, apoptosis and DNA repair [42-48]. In relation to MAPK signalling, previous studies have shown that MAPK activity is required for PanIN formation and occurs early during the pancreatic transformation [49]. Similarly, SMADs have been identified as proteins that transduce the upstream signalling from TGF-Beta superfamily, thereby influencing cell proliferation, differentiation, and apoptosis $[50,51]$. Finally, both the signalling pathways mediated ErbB1 and c-MET have been described for their associations with a more aggressive phenotype and poor prognosis in patients with pancreatic adenocarcinoma [52, 53].

Finally, the interactions network between miRNAs differentially expressed in tumour compared to normal tissues from PDAC corroborated the noteworthy complexity feature of the network and reflected the complexity of the disease at issue. Due to its size, this network has been clustered into five highly cohesive groups of miRNAs. The first cluster seemed to have a relevant role since a subset of miRNAs belonging to this cluster emerged as the top-five ranked for at least one topological index. Pathway analyses emphasized the importance of the signalling events mediated by TGF-beta in PDAC.

Disruption of normal TGF-beta pathway has been implicated in the pathogenesis and progression of pancreatic cancer, where it may play a dual effect as tumour suppressor and as a tumour promoter in normal and malignant cells, respectively [50]. In details, our finding showed that TGFbeta pathway was influenced by several miRNAs (miR-21, miR-214, miR146a, miR-30b, miR-195, miR503, miR181a$\left.a^{*}-b-c^{*}-d\right)$ on 3 main genes (TFGBR2, SMAD3, SMAD4) in PDAC.

Networks analysis allowed to identify three main subgroups of miRNAs also in PVAC. Overall, the miRNAs and the signalling pathways closed to the tumour network did not correspond to those highlighted in the diseased network wired for PDAC in PVAC. Conversely, a partial overlap in relation to TGF-beta signalling events emerged from enrichment analysis based on either miRNAs shared between normal and tumour network and on those significantly altered in tumour compared to normal tissues samples in PVAC.

In details, the three miRNAs present only in the diseased network (miR-199b-5p, miR-323a -3p, miR-563) regulated a number of target genes involved in the LICAM1 and AXON guidance signalling pathways. Other authors reported that the increase of LICAM1 expression levels were associated with the chemo resistance and migratory phenotype of pancreatic cancer cells, and showed the significant correlation with the degree of perineural invasion of the tumour and the clinical course of patients $[54,55]$. Similarly, the signals transmitted along the axons have been recently confirmed to be associated with diffusion and metastasis in pancreatic cancer [56, 57].

Among the miRNAs shared between the two networks, we found that the only common correlation was that between $\mathrm{miR}-1228^{*}$ and $\mathrm{miR}-1280$; although Schopman et al. showed that the sequence annotated as miR-1280 is likely to be a fragment of a tRNA [58], these two miRNAS were likely to initiate and drive the correlation chains for normal and tumour tissues, respectively. In relation to $\mathrm{miR}-889$, even if this miRNA did not target any genes associated with pancreatic cancer, its changes in either direction and neighbouring in the two networks may be the sign of an important change in the functional targeting between healthy and tumour tissues. Conversely, miR-103 was the only miRNA among those with a high fold-change value that targeted genes associated with pancreatic cancer; it was in a prominent topological position in normal network, whereas it was the only miRNA to be highly expressed and is likely to drive the longest chain of consecutive inverse correlations in the tumour network. We found that miR-103 influenced, TFG-beta pathways (TGFBR2, APC, CDH1, CREBBP, EP300, SMAD3, TSC2), altered TGF-beta SMAD dependent signalling (TGFBR2, SMAD3, FBXW7), and E-cadherin signalling events $(\mathrm{CDH} 1)$ in PVAC.

Noteworthy, miR-103 seemed to have a peculiar role even in PDAC, where it was an important hub of the interactions network between miRNAs differentially expressed in tumours compared to normal tissues; therefore, even if it did not directly modulate many genes, it might exert a relevant influence to the target genes of its neighbour miRNAs, most of which belong to the cluster 2 of the network. The shared centrality of miR-103 in the two cancer entities was accompanied by an overlap in the signalling pathways associated with this miRNA. As uncovered by enrichment analysis, miR-103 influenced the signalling pathway mediated by TFG-Beta in PDAC and PVAC. However, while in PDAC more miRNAs connected to miR-103 regulated specific target genes, in PVAC miR103 exerted its direct influence on a larger number of genes.

In relation to these genes, recent studies have revealed a molecular association between TGF-beta/ SMADs signalling pathways and tuberin (TSC2), a tumour suppressor gene involved in cell growth and differentiation, and in cell cycle progression [59]. SMADs were also reported to interact with CBP and p300, proteins having histone acetyl transferase and histone deacetylase activities, and to play an important role in cell proliferation and differentiation [60]. The association of SMAD2 and SMAD3 with APC gene was reported to contribute as antagonist of the events mediated by TGF-beta [61]. Within the SMAD signal transduction pathway, FBXW7 gene enhances 
TGF $\beta$-dependent transcription by inactivating the repressor transcriptional repressor TGIF1 [62-64]. Furthermore, CDH1, the adhesion molecule involved in cell-to-cell cohesion, cell-to-cell recognition, and epithelial polarity, is known to be regulated by TGF $\beta$ in pancreatic ductal adenocarcinoma [65]. It is involved in tumour progression and in prognosis of patients with pancreatic cancer [66-71].

Evaluation of interactions network between miRNAs differentially expressed in tumour compared to normal pancreatic tissues from PVAC highlighted that other two miRNAs (miR-200c ${ }^{*}$ and miR-29b-2*) contributed with miR-103 to enriching TFG-beta and altered TGF-beta SMAD dependent, signalling events E-cadherin, pathway and signals mediated by p53 and p21. Once again, data showed an overlap in the regulation of TGF beta pathway. However, it must be stressed that the signalling events at issue were regulated by different miRNAs and in turn by different target genes in PVAC compare to PDAC.

Noteworthy, another peculiarity in PVAC concerned the topological organization of miRNAs forming two closed groups in the normal network. Since these closed groups did not persist in the diseased network, they might be the sign of an important change in functional targeting between healthy and tumour tissues. Furthermore, it was hypothesized that these closed groups can identify "functional collaborators": genes and biological processes regulated by miRNAs can be associated with each other; alternatively it was possible to speculate that the effects of a miRNA on target genes and their respective signalling pathways occur according to miRNA expression, which in turn may depend on the correlation between the expression levels of miRNAs in the closed groups [72-74]. In details, miR-548b-3p of the first closed group regulated DICER1, involved in the biogenesis of miRNAs themselves;Recently, a potential oncogenic role for DICER1 in pancreatic cancer initiation was also reported [75-77]. On the other hand several genes were influenced by miR-140-5p of the second closed group. These genes significantly enriched the signalling events mediated by p53, p21 and E-cadherin. The importance of these pathways in pancreatic carcinogenesis are well documented [68, 69, 72, 78, 79].

In relation to the pathways influenced by miR-140$5 \mathrm{p}$, a difference between PDAC and PVAC emerged:miR$140-5 p$ acts independently and directly in PVAC, whereas its neighbour miR-27b-3p regulates of the same signalling events in PDAC.

In addition, we found that the signalling pathways mediated by miR-140-5p were influenced also by miR199b-5p in PDAC: its three neighbours (miR-196a, let7d*, miR-520a-3p) regulated several target genes (TP53, CDH1, TSC2, CREBBP, TGFBR2, SMAD3, SMAD4) involved in these signalling events.

On the other hand, miR-1254 exemplified the case of a miRNA involved in the same signalling pathways in the two entities of pancreatic cancer, albeit thought different neighbours influencing different target genes. Indeed, either ERCC4 and EP300, targeted by let$7 \mathrm{~g}$ and miR-200 $\mathrm{c}^{*}$ in PDAC in PVAC respectively, influenced the DNA damage repair pathway. Evidences have revealed that ERCC4 expression levels are

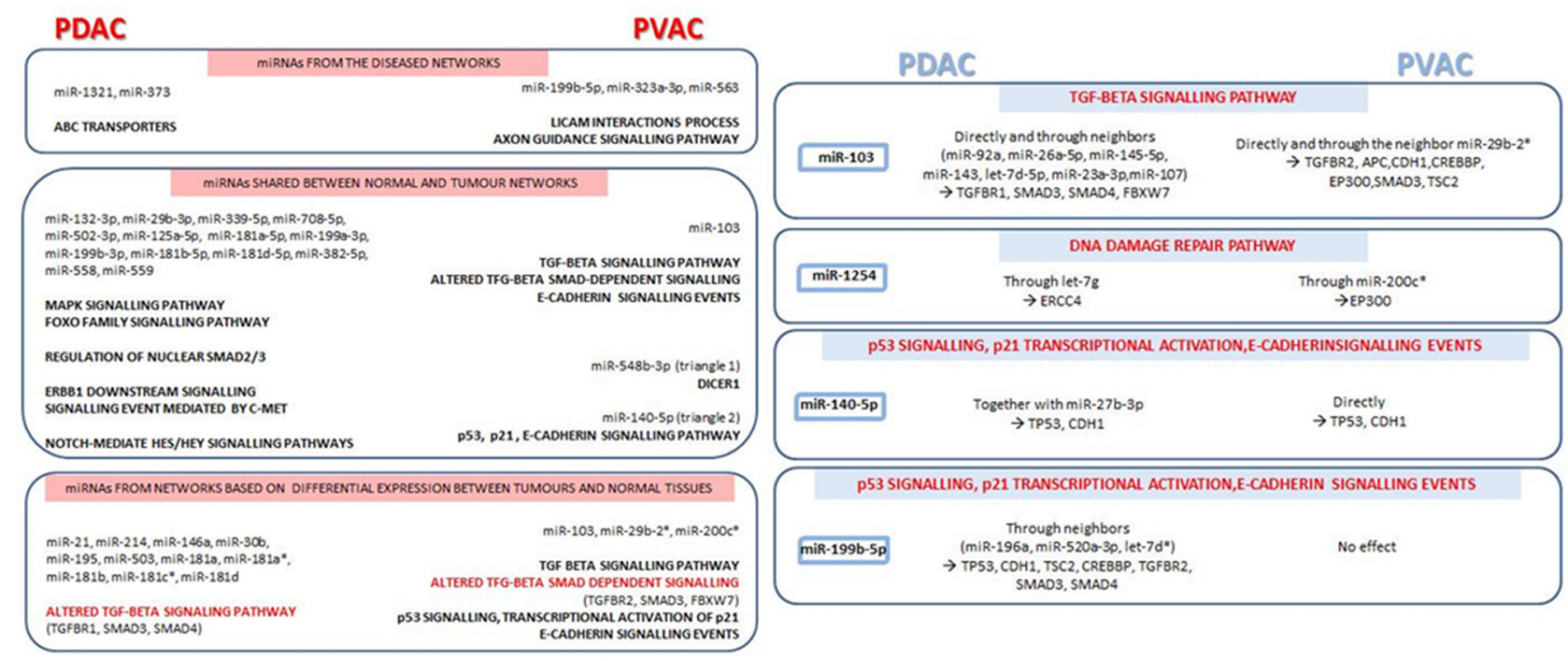

Figure 6: Main signalling pathways, together with miRNAs and respective target genes, emerged from microRNA co-expression networks in pancreatic ductal adenocarcinoma (PDAC) compared to Vater's papilla adenocarcinoma (PVAC). Shared signalling events, regulated by different miRNAs and/or genes in PDAC and PVAC, are highlighted in red. 
correlated with cancer risk, progression, response to chemotherapy, and clinical outcome of different tumours, such as head and neck cancer, suggesting that altered ERCC4 expression may lead to altered DNA repair capacity, thereby modulating cancer susceptibility [80]. Similarly, EP300 is a transcriptional co-activator that mediates many transcriptional events including DNA repair [81].

Finally, the enrichment analysis was performed by using the 27 miRNAs significantly altered in matched pairs of tissues from PDAC compared to those from PVAC, in order to predict a role for miRNAs in distinguishing between PDAC and PVAC. A part from conforming the involvement of miRNAs in some of the pathways previously associated with PDAC or PVAC, our findings uncovered the regulation of the Wnt and the Hedgehog signalling pathways. Wnt signalling events were reported in the literature for their association with the carcinogenesis and progression of pancreatic cancer [82]. Similarly, activation of Hedgehog signalling pathway influences cell proliferation and cell cycle and thus has a role for initiation of pancreatic cancer either alone or in a K-Ras dependent way [83, 84].

In general, PDAC was characterized by a more consistent miRNA alteration compared to PVAC, and a more complex interactions networks were found either for miRNAs connected in tumour tissues and for those altered in tumours compared to normal pancreatic samples. However, with few exceptions especially in relation to the signalling events regulated by miRNAs connected only in the tumour networks and for those emerged from miRNAs shared between tumour and normal networks, our findings show a good overlap between the signalling pathways influenced by miRNAs in the two tumour types. Nevertheless, the shared signalling pathways were regulated by different miRNAs and/or genes in PDAC and PVAC (Figure 6).

Overall our findings reflect the specificity of miRNAs expression patter within different entities of pancreatic cancer, and suggest a role for these molecules in the regulation of a limited core signalling pathways in the biology landscape of PDAC and PVAC.

Father studies are needed to corroborate our data from co-expression networks. In details, the biological effect of specific miRNAs should be functionally validated in vitro to verify the interaction between miRNA and mRNA target, their co-expression, and their effect on the predicted protein expression up to the alteration of respective signalling pathways.

\section{MATERIALS AND METHODS}

\section{MiRNA profiling datasets}

The main cohort of this study is made of two groups of patients, 9 PDAC and 4 PVAC, enclosed in our previous miRNAs expression profiling study on matched-pairs of normal and tumour tissues samples from 17 patients with different histological types of pancreatobiliary cancer (including, apart from PDAC and PVAC, adenocarcinoma arising from the biliary epithelium) [85].

Tissue samples were obtained from patients who underwent pancreatic resection for PDAC or PVAC, and before any chemotherapy had been initiated. In details, specimens were harvested during surgery after extemporaneous anatomopathological test for evaluation of both the tumour and the adjacent non-affected pancreatic tissue which were then taken and stored separately. In addition, later pathological evaluations ascertained the origin from duodenal mucosa for all the PVAC samples.

Gene expression was profiled with Affymetrix GeneChip miRNA 2.0 Arrays and made available from ArrayExpress (E-MTAB-753). Symbols of miRNAs were converted to their most recent forms and annotated with their MIMAT IDs using the aliases tracking system provided by miRBase (Supplementary Information \#Sheet B).

An external dataset (E-GEOD-60978), made of 52 PDAC and 6 normal tissues profiled by Agilent 031181 Unrestricted Human miRNA V16.0 Microarray 030840 platform, was used to crosscheck the evidences of expression correlation found in this work. No public datasets of miRNA expression profiles of PVAC were found, therefore a similar analysis for our PVAC counterpart could not be made.

\section{Statistical analysis}

In order to identify differentially expressed miRNAs between paired normal and PDAC/PVAC tumour tissues, paired $t$-tests were performed controlling for false discovery rate (fdr) allowing us to rank miRNAs according to their p-values. In each cohort, correlations between miRNAs expression were estimated using Spearman coefficient, in order to account for non-normal data distribution. These correlation matrices were then used for bioinformatics analyses. A p-value of 0.05 was considered for statistical significance. All analyses were performed using SAS Release 9.1 (SAS Institute, Cary, NC, USA).

\section{Geometric and topological analysis}

Similar to the analysis workflow followed in [85] and [86], we built undirected and weighted graphs of miRNA-to-miRNA based on correlation data. Nodes and edges of graphs represented miRNAs and their correlation values, if significant, respectively. Edges were not oriented since correlation is a symmetric measure, and were weighted with the Spearman coefficient. Initially, graphs were drawn separately for normal and tumour tissues. Then, only differentially expressed miRNAs between both tissues for both tumour entities were considered. Networks were drawn and analysed by Cytoscape 3.1.0 [87] and by a custom standalone tool written in $\mathrm{CH}$ and built over the library NodeXL 1.0.1.317. 


\section{Global geometries}

Graphs connectivity, reachability and cohesiveness were measured by well-known global topological indices. We first counted the number of connected components, which is the number of groups of nodes that are pairwise connected. A lower number of connected components suggest a stronger connectivity. Then, we measured the diameter, meant as the longest among all the shortest paths of a network, and the characteristic path length, also known as the average shortest path length, which gives the expected distance between two connected nodes. We further considered the network centralization, which is 1 if a network resembles a star or 0 if it is decentralized, the average number of neighbours, which indicates the average connectivity of a node, and its normalized version: the network density. The density is a value between 0 and 1. It shows how densely a network is populated with edges. A network that contains no edges and solely isolated nodes has a density of 0 . In contrast, the density of a clique is $1[88,89]$. Finally, we considered the network heterogeneity that reflects the tendency of a network to contain central and highly connected nodes, also known as hubs.

\section{Local topological metrics}

Critical miRNAs were recognized because of their topological measures: degree, betweenness, closeness and clustering coefficient [90-92]. All of them are based on the enumeration of links or shortest paths, whose length is calculated by summing the inverse weights of the traversing edges. The idea is that highly correlated miRNAs minimize the distance between nodes. Thus, while degree centrality relies on the fact that important nodes are those with the largest number of ties to other nodes, the betweenness index measures the influence a node has over the indirect correlation between even distant not-neighbour nodes. Closeness highlights nodes that are particularly 'close' to other nodes, or 'reachable' from other nodes. The shorter the geodesic distance from a node to other nodes, the higher its closeness centrality. On the contrary, the clustering coefficient measures the degree to which miRNAs tend to cluster together. Scatter plots summarizing the distributions of local topological indices are available in Supplementary Figure 1.

\section{Cluster-based functional enrichment analysis}

Important groups of miRNAs were identified by the Molecular Complex Detection (MCODE) clustering algorithm [93]. Weighting each miRNA by the local neighbourhood density and outward traversal from locally dense seed miRNAs, we isolated several dense regions that shared molecular targets and putatively cooperated to the fulfilment of a common biological process.

MirTarBase [94] and MirWalk [95] were queried (April 2015) to get experimentally validated target genes of selected miRNAs. Clashes of names and aliases were resolved by querying miRBase [96]. Target genes underwent functional enrichment analysis against the Gene Ontology FAT sub-set. Results obtained with DAVID [97] and ToppGene [98] web services were crosschecked with Babelomics [99] and considered if Bonferroni-corrected significance levels did not exceed 5\%.

\section{ACKNOWLEDGMENTS}

None.

\section{CONFLICTS OF INTEREST} declare.

Authors do not have any conflicts of interest to

\section{FUNDING}

The study was supported by the Italian Ministry of Health grants (RC1603GA33, RC1703GA33) to the Division of Gastroenterology, IRCCS "Casa Sollievo della Sofferenza" Hospital, San Giovanni Rotondo (FG), Italy and by the " $5 \times 1000$ " voluntary contribution.

\section{REFERENCES}

1. Siegel RL, Miller KD, Jemal A. Cancer statistics, 2016. CA Cancer J Clin. 2016; 66:7-30.

2. Yabar CS, Winter JM. Pancreatic cancer: a review. Gastroenterol Clin North Am. 2016; 45:429-45.

3. Chakraborty S, Baine MJ, Sasson AR, Batra SK. Current status of molecular markers for early detection of sporadic pancreatic cancer. Biochim Biophys Acta. 2011; 1815:44-64.

4. Wagner M, Redaelli C, Lietz M, Seiler CA, Friess H, Buchler MW. Curative resection is the single most important factor determining outcome in patients with pancreatic adenocarcinoma. Br J Surg. 2004; 91:586-94.

5. Ahn DH, Bekaii-Saab T. Ampullary cancer: an overview. Am Soc Clin Oncol Educ Book. 2014.

6. Askew J, Connor S. Review of the investigation and surgical management of resectable ampullary adenocarcinoma. HPB (Oxford). 2013; 15:829-38.

7. Klein F, Jacob D, Bahra M, Pelzer U, Puhl G, Krannich A, Andreou A, Gül S, Guckelberger O. Prognostic factors for long-term survival in patients with ampullary carcinoma: the results of a 15-year observation period after pancreaticoduodenectomy. HPB Surg. 2014; 2014:970234.

8. Esquela-Kerscher A, Slack FJ. Oncomirs-microRNAs with a role in cancer. Nat Rev Cancer. 2006; 6:259-69.

9. Iorio $\mathrm{MV}$, Croce CM. microRNA involvement in human cancer. Carcinogenesis. 2012; 33:1126-33.

10. Bloomston M, Frankel WL, Petrocca F, Volinia S, Alder H, Hagan JP, Liu CG, Bhatt D, Taccioli C, Croce CM. 
MicroRNA expression patterns to differentiate pancreatic adenocarcinoma from normal pancreas and chronic pancreatitis. JAMA. 2007; 297:1901-8.

11. Lee EJ, Gusev Y, Jiang J, Nuovo GJ, Lerner MR, Frankel WL, Morgan DL, Postier RG, Brackett DJ, Schmittgen TD. Expression profiling identifies microRNA signature in pancreatic cancer. Int J Cancer. 2007; 120:1046-54.

12. Mardin WA, Mees ST. MicroRNAs: novel diagnostic and therapeutic tools for pancreatic ductal adenocarcinoma? Ann Surg Oncol. 2009; 16:3183-9.

13. Jamieson NB, Morran DC, Morton JP, Ali A, Dickson EJ, Carter CR, Sansom OJ, Evans TR, McKay CJ, Oien KA. MicroRNA molecular profiles associated with diagnosis, clinicopathologic criteria, and overall survival in patients with resectable pancreatic ductal adenocarcinoma. Clin Cancer Res. 2012; 18:534-45.

14. van Dam S, Võsa U, van der Graaf A, Franke L, de Magalhães JP. Gene co-expression analysis for functional classification and gene-disease predictions. Brief Bioinform. 2017.

15. Seux M, Iovanna J, Dagorn JC, Dusetti NJ. MicroRNAs in pancreatic ductal adenocarcinoma: new diagnostic and therapeutic clues. Pancreatology. 2009; 9:66-72.

16. Steele CW, Oien KA, McKay CJ, Jamieson NB. Clinical potential of microRNAs in pancreatic ductal adenocarcinoma. Pancreas. 2011; 40:1165-71.

17. Diab M, Muqbil I, Mohammad RM, Azmi AS, Philip PA. The role of microRNAs in the diagnosis and treatment of pancreatic adenocarcinoma. J Clin Med. 2016; 5:59.

18. Schultz NA, Werner J, Willenbrock H, Roslind A, Giese N, Horn T, Wøjdemann M, Johansen JS. MicroRNA expression profiles associated with pancreatic adenocarcinoma and ampullary adenocarcinoma. Mod Pathol. 2012; 25:1609-22.

19. Zhang B, Pan X, Cobb GP, Anderson TA. microRNAs as oncogenes and tumor suppressors. Dev Biol. 2007; 302:1-12.

20. Furukawa T, Sunamura M, Horii A. Molecular mechanisms of pancreatic carcinogenesis. Cancer Sci. 2006; 97:5.

21. Prenzel KL, Warnecke-Eberz U, Brabender J, Baldus SE, Bollschweiler E, Gutschow CA, Drebber U, Hoelscher AH, Schneider PM. Differential c-erbB-1 and c-erbB-2 mRNA expression in cancer of the pancreas compared with cancer of the papilla of Vater. World J Gastroenterol. 2006; 12:437-42.

22. Esposito I, Friess H, Büchler MW. Carcinogenesis of cancer of the papilla and ampulla: pathophysiological facts and molecular biological mechanisms. Langenbecks Arch Surg. 2001; 386:163-71.

23. di Mola FF, di Sebastiano P. Pain and pain generation in pancreatic cancer. Langenbecks Arch Surg. 2008; 393:919-22.

24. D'Haese JG, Hartel M, Demir IE, Hinz U, Bergmann F, Büchler MW, Friess H, Ceyhan GO. Pain sensation in pancreatic diseases is not uniform: the different facets of pancreatic pain. World J Gastroenterol. 2014; 20:9154-61.

25. Zhao Z, Zhao Q, Warrick J, Lockwood CM, Woodworth A, Moley KH, Gronowski AM. Circulating microRNA miR323-3p as a biomarker of ectopic pregnancy. Clin Chem. 2012; 58:896-905.

26. Tiwari AK, Sodani K, Dai CL, Ashby CR Jr, Chen ZS. Revisiting the $\mathrm{ABCs}$ of multidrug resistance in cancer chemotherapy. Curr Pharm Biotechnol. 2011; 12:570-94.

27. Chen Z, Shi T, Zhang L, Zhu P, Deng M, Huang C, Hu T, Jiang L, Li J. Mammalian drug efflux transporters of the ATP binding cassette (ABC) family in multidrug resistance: a review of the past decade. Cancer Lett. 2016; 370:153-64.

28. Bai J, Sata N, Nagai H. Gene expression analysis for predicting gemcitabine sensitivity in pancreatic cancer patients. HPB (Oxford). 2007; 9:150-5.

29. Mohelnikova-Duchonova B, Brynychova V, Oliverius M, Honsova E, Kala Z, Muckova K, Soucek P. Differences in transcript levels of $\mathrm{ABC}$ transporters between pancreatic adenocarcinoma and nonneoplastic tissues. Pancreas. 2013; 42:707-16.

30. Konig J, Hartel M, Nies AT, Martignoni ME, Guo J, Buchler MW, Friess H, Keppler D. Expression and localization of human multidrug resistance protein (ABCC) family members in pancreatic carcinoma. Int J Cancer. 2005; 115:359-67.

31. Hagmann W, Jesnowski R, Faissner R, Guo C, Lohr JM. ATP-binding cassette $\mathrm{C}$ transporters in human pancreatic carcinoma cell lines. Upregulation in 5-fluorouracilresistant cells. Pancreatology. 2009; 9:136-44.

32. Hagmann W, Jesnowski R, Lohr JM. Interdependence of gemcitabine treatment, transporter expression and resistance in human pancreatic carcinoma cells. Neoplasia. $2010 ; 12: 740-7$.

33. Nambaru PK, Hübner T, Köck K, Mews S, Grube M, Payen L, Guitton J, Sendler M, Jedlitschky G, Rimmbach C, Rosskopf D, Kowalczyk DW, Kroemer HK, et al. Drug efflux transporter multidrug resistance-associated protein 5 affects sensitivity of pancreatic cancer cell lines to the nucleoside anticancer drug 5-fluorouracil. Drug Metab Dispos. 2011; 39:132-9.

34. Radtke F, Raj K. The role of Notch in tumorigenesis: oncogene or tumour suppressor? Nat Rev Cancer. 2003; 3:756-67.

35. Ma J, Xia J, Miele L, Sarkar FH, Wang Z. Notch signaling pathway in pancreatic cancer progression. Pancreat Disord Ther. 2013; 3:1000114.

36. Büchler P, Gazdhar A, Schubert M, Giese N, Reber HA, Hines OJ, Giese T, Ceyhan GO, Müller M, Büchler MW, Friess H. The Notch signaling pathway is related to neurovascular progression of pancreatic cancer. Ann Surg. 2005; 242:791-800.

37. Miyamoto Y, Maitra A, Ghosh B, Zechner U, Argani P, Iacobuzio-Donahue CA, Sriuranpong V, Iso T, Meszoely 
IM, Wolfe MS, Hruban RH, Ball DW, Schmid RM, Leach SD. Notch mediates TGF alpha-induced changes in epithelial differentiation during pancreatic tumorigenesis. Cancer Cell. 2003; 3:565-76.

38. Wang Z, Zhang Y, Li Y, Banerjee S, Liao J, Sarkar FH. Down-regulation of Notch-1 contributes to cell growth inhibition and apoptosis in pancreatic cancer cells. Mol Cancer Ther. 2006; 5:483-93.

39. Wang Z, Zhang Y, Banerjee S, Li Y, Sarkar FH. Inhibition of nuclear factor kappa b activity by genistein is mediated via Notch-1 signaling pathway in pancreatic cancer cells. Int J Cancer. 2006; 118:1930-6.

40. Wang Z, Li Y, Banerjee S, Kong D, Ahmad A, Nogueira V, Hay N, Sarkar FH. Down-regulation of Notch-1 and Jagged-1 inhibits prostate cancer cell growth, migration and invasion, and induces apoptosis via inactivation of Akt, mTOR, and NF-kappaB signaling pathways. J Cell Biochem. 2010; 109:726-36.

41. Plentz R, Park JS, Rhim AD, Abravanel D, Hezel AF, Sharma SV, Gurumurthy S, Deshpande V, Kenific C, Settleman J, Majumder PK, Stanger BZ, Bardeesy N. Inhibition of gamma-secretase activity inhibits tumor progression in a mouse model of pancreatic ductal adenocarcinoma. Gastroenterology. 2009; 136:1741-9.

42. Zhang Y, Gan B, Liu D, Paik JH. FoxO family members in cancer. Cancer Biol Ther. 2011; 12:253-9.

43. Accili D, Arden KC. FoxOs at the crossroads of cellular metabolism, differentiation and transformation. Cell. 2004; 117:421-6.

44. Martinez-Gac L, Marques M, Garcia Z, Campanero MR, Carrera AC. Control of cyclin G2 mRNA expression by forkhead transcription factors: novel mechanism for cell cycle control by phosphoinositide-3-kinase and forkhead. Mol Cell Biol. 2004; 24:2181-9.

45. Furukawa-Hibi Y, Yoshida-Araki K, Ohta T, Ikeda K, Motoyama N. FOXO forkhead transcription factors induce $\mathrm{G}(2)-\mathrm{M}$ checkpoint in response to oxidative stress. J Biol Chem. 2002; 277:26729-32.

46. Tran H, Brunet A, Grenier JM, Datta SR, Fornace AJ Jr, DiStefano PS, Chiang LW, Greenberg ME. DNA repair pathway stimulated by the forkhead transcript3ion factor FOXO3a through the Gadd45 protein. Science. 2002; 296:530-4.

47. Tang TT, Dowbenko D, Jackson A, Toney L, Lewin DA, Dent AL, Lasky LA. The forkhead transcription factor AFX activates apoptosis by induction of the BCL-6 transcriptional repressor. J Biol Chem. 2002; 277:14255-65.

48. Roy SK, Chen Q, Fu J, Shankar S, Srivastava RK. Resveratrol inhibits growth of orthotopic pancreatic tumors through activation of FOXO transcription factors. PLoS One. 2011; 6:e25166.

49. Collisson EA, Trejo CL, Silva JM, Gu S, Korkola JE, Heiser LM, Charles RP, Rabinovich BA, Hann B, Dankort D, Spellman PT, Phillips WA, Gray JW, et al. A central role for
$\mathrm{RAF} \rightarrow \mathrm{MEK} \rightarrow$ ERK signaling in the genesis of pancreatic ductal adenocarcinoma. Cancer Discov. 2012; 2:685-93.

50. Truty MJ, Urrutia R. Basics of TGF-beta and pancreatic cancer. Pancreatology. 2007; 7:423-35.

51. Singh P, Srinivasan R, Wig JD. The Smad family and its role in pancreatic cancer. Indian J Cancer. 2011; 48:351-60.

52. Ueda S, Ogata S, Tsuda H, Kawarabayashi N, Kimura M, Sugiura Y, Tamai S, Matsubara O, Hatsuse K, Mochizuki $\mathrm{H}$. The correlation between cytoplasmic overexpression of epidermal growth factor receptor and tumor aggressiveness: poor prognosis in patients with pancreatic ductal adenocarcinoma. Pancreas. 2004; 29:e1-8.

53. Di Renzo MF, Poulsom R, Olivero M, Comoglio PM, Lemoine NR. Expression of the Met/hepatocyte growth factor receptor in human pancreatic cancer. Cancer Res. 1995; 55:1129-38.

54. Ben QW, Wang JC, Liu J, Zhu Y, Yuan F, Yao WY, Yuan YZ. Positive expression of L1-CAM is associated with perineural invasion and poor outcome in pancreatic ductal adenocarcinoma. Ann Surg Oncol. 2010; 17:2213-21.

55. Tsutsumi S, Morohashi S, Kudo Y, Akasaka H, Ogasawara H, Ono M, Takasugi K, Ishido K, Hakamada K, Kijima H. L1 Cell adhesion molecule (L1CAM) expression at the cancer invasive front is a novel prognostic marker of pancreatic ductal adenocarcinoma. J Surg Oncol. 2011; 103:669-73.

56. Biankin AV, Waddell N, Kassahn KS, Gingras MC, Muthuswamy LB, Johns AL, Miller DK, Wilson PJ, Patch AM, Wu J, Chang DK, Cowley MJ, Gardiner BB, et al. Pancreatic cancer genomes reveal aberrations in axon guidance pathway genes. Nature. 2012; 491:399-405.

57. Göhrig A, Detjen KM, Hilfenhaus G, Körner JL, Welzel M, Arsenic R, Schmuck R, Bahra M, Wu JY, Wiedenmann $\mathrm{B}$, Fischer C. Axon guidance factor SLIT2 inhibits neural invasion and metastasis in pancreatic cancer. Cancer Res. 2014; 74:1529-40.

58. Schopman NC, Heynen S, Haasnoot J, Berkhout B. A miRNA-tRNA mix-up: tRNA origin of proposed miRNA. RNA Biol. 2010; 7:573-6.

59. Birchenall-Roberts $\mathrm{MC}, \mathrm{Fu} \mathrm{T}$, Bang OS, Dambach M, Resau JH, Sadowski CL, Bertolette DC, Lee HJ, Kim SJ, Ruscetti FW. Tuberous sclerosis complex 2 gene product interacts with human SMAD proteins. A molecular link of two tumor suppressor pathways. J Biol Chem. 2004; 279:25605-13.

60. Iyer NG, Ozdag H, Caldas C. p300/CBP and cancer. Oncogene. 2004; 23:4225-31.

61. Stroschein SL, Bonni S, Wrana JL, Luo K. Smad3 recruits the anaphase-promoting complex for ubiquitination and degradation of SnoN. Genes Dev. 2001; 15:2822-36.

62. Bengoechea-Alonso MT, Ericsson J. Tumor suppressor Fbxw7 regulates TGF $\beta$ signaling by targeting TGIF1 for degradation. Oncogene. 2010; 29:5322-8. 
63. Pessah M, Prunier C, Marais J, Ferrand N, Mazars A, Lallemand F, Gauthier JM, Atfi A. c-Jun interacts with the corepressor TG-interacting factor (TGIF) to suppress Smad2 transcriptional activity. Proc Natl Acad Sci U S A. 2001; 98:6198-203.

64. Wotton D, Massagué J. Smad transcriptional corepressors in TGF beta family signalling. Curr Top Microbiol Immunol. 2001; 254:145-64.

65. Geng MM, Ellenrieder V, Wallrapp C, Muller-Pillasch F, Sommer G, Adler G, Gress TM. Use of representational difference analysis to study the effect of TGFB on the expression profile of a pancreatic cancer cell line. Genes Chromosomes Cancer. 1999; 26:70-9.

66. Jeanes A, Gottardi CJ, Yap AS. Cadherins and cancer: how does cadherin dysfunction promote tumor progression? Oncogene. 2008; 27:6920-9.

67. Karayiannakis AJ, Syrigos KN, Polychronidis A, Simopoulos C. Expression patterns of alpha-, beta- and gamma-catenin in pancreatic cancer: correlation with E-cadherin expression, pathological features and prognosis. Anticancer Res. 2001; 21:4127-34.

68. Li YJ, Ji XR. Relationship between expression of E-cadherin-catenin complex and clinicopathologic characteristics of pancreatic cancer. World J Gastroenterol. 2003; 9:368-72.

69. Joo YE, Rew JS, Park CS, Kim SJ. Expression of E-cadherin, alpha- and beta-catenins in patients with pancreatic adenocarcinoma. Pancreatology. 2002; 2:129-37.

70. Chen HC, Chu RY, Hsu PN, Hsu PI, Lu JY, Lai KH, Tseng HH, Chou NH, Huang MS, Tseng CJ, Hsiao M. Loss of E-cadherin expression correlates with poor differentiation and invasion into adjacent organs in gastric adenocarcinomas. Cancer Lett. 2003; 201:97-106.

71. Winter JM, Ting AH, Vilardell F, Gallmeier E, Baylin SB, Hruban RH, Kern SE, Iacobuzio-Donahue CA. Absence of E-cadherin expression distinguishes noncohesive from cohesive pancreatic cancer. Clin Cancer Res. 2008; $14: 412-8$.

72. Petkov PM, Graber JH, Churchill GA, DiPetrillo K, King BL, Paigen K. Evidence of a large-scale functional organization of Mammalian chromosomes. PLoS Biol. 2007; 5:e127.

73. Yang Y, Han L, Yuan Y, Li J, Hei N, Liang H. Gene co-expression network analysis reveals common systemlevel properties of prognostic genes across cancer types. Nat Commun. 2014; 5:3231.

74. Nepusz T, Yu H, Paccanaro A. Detecting overlapping protein complexes in protein-protein interaction networks. Nat Meth. 2012; 9:471-2.

75. Sahasrabuddhe NA, Huang TC, Kumar P, Yang Y, Ghosh B, Leach SD, Chaerkady R, Pandey A. Ablation of Dicer leads to widespread perturbation of signaling pathways. Biochem Biophys Res Commun. 2015; 463:389-94.
76. Morris JP 4th, Greer R, Russ HA, von Figura G, Kim GE, Busch A, Lee J, Hertel KJ, Kim S, McManus M, Hebrok M. Dicer regulates differentiation and viability during mouse pancreatic cancer initiation. PLoS One. 2014; 9:e95486.

77. Wang YJ, McAllister F, Bailey JM, Scott SG, Hendley AM, Leach SD, Ghosh B. Dicer is required for maintenance of adult pancreatic acinar cell identity and plays a role in Krasdriven pancreatic neoplasia. PLoS One. 2014; 9:e113127.

78. Riley T, Sontag E, Chen P, Levine A. Transcriptional control of human p53-regulated genes. Nat Rev Mol Cell Biol. 2008; 9:402-12.

79. Vogelstein B, Lane D, Levine AJ. Surfing the p53 network. Nature. 2000; 408:307-10.

80. Yu H, Liu Z, Huang YJ, Yin M, Wang LE, Wei Q. Association between single nucleotide polymorphisms in ERCC4 and risk of squamous cell carcinoma of the head and neck. PLoS One. 2012; 7:e41853.

81. Hasan S, Hassa PO, Imhof R, Hottiger MO. Transcription coactivator p300 binds PCNA and may have a role in DNA repair synthesis. Nature. 2001; 410:387-91.

82. Zhang Y, Morris JP 4th, Yan W, Schofield HK, Gurney A, Simeone DM, Millar SE, Hoey T, Hebrok M, Pasca di Magliano M. Canonical wnt signaling is required for pancreatic carcinogenesis. Cancer Res. 2013; 73:4909-22.

83. Pasca di Magliano M, Sekine S, Ermilov A, Ferris J, Dlugosz AA, Hebrok M. Hedgehog/Ras interactions regulate early stages of pancreatic cancer. Genes Dev. 2006; 20:3161-73.

84. Thayer SP, di Magliano MP, Heiser PW, Nielsen CM, Roberts DJ, Lauwers GY, Qi YP, Gysin S, Fernández-del Castillo C, Yajnik V, Antoniu B, McMahon M, Warshaw AL, Hebrok M. Hedgehogs is an early and late mediator of pancreatic cancer tumorigenesis. Nature. 2003; 425:851-6.

85. Piepoli A, Tavano F, Copetti M, Mazza T, Palumbo O, Panza A, di Mola FF, Pazienza V, Mazzoccoli G, Biscaglia G, Gentile A, Mastrodonato N, Carella M, et al. MiRNA expression profiles identify drivers in colorectal and pancreatic cancers. PLoS One. 2012; 7:e33663.

86. Mazzoccoli G, Tomanin R, Mazza T, D’Avanzo F, Salvalaio M, Rigon L, Zanetti A, Pazienza V, Francavilla M, Giuliani F, Vinciguerra M, Scarpa M. Circadian transcriptome analysis in human fibroblasts from Hunter syndrome and impact of iduronate-2-sulfatase treatment. BMC Med Genomics. 2013; 6:37.

87. Shannon P, Markiel A, Ozier O, Baliga NS, Wang JT, Ramage D, Amin N, Schwikowski B, Ideker T. Cytoscape: a software environment for integrated models of biomolecular interaction networks. Genome Res. 2003; 13:2498-504.

88. Mazza T, Romanel A, Jordán F. Estimating the divisibility of complex biological networks by sparseness indices. Brief Bioinform. 2010; 11:364-74.

89. Menniti S, Castagna E, Mazza T. Estimating the global density of graphs by a sparseness index. Appl Math Comput. 2013; 224:346-57. 
90. Barabasi AL. Network science. Philos Trans A Math Phys Eng Sci. 2013; 371:20120375.

91. Sabidussi G. The centrality index of a graph. Psychometrika. 1966; 31:581-603.

92. Mazza T, Prandi D. Stability analysis of biological network topologies during stochastic simulation. SIMUTools 2011 Proceedings of the 4th International ICST Conference on Simulation Tools and Techniques, 51-6.

93. Bader GD, Hogue CW. An automated method for finding molecular complexes in large protein interaction networks. BMC Bioinformatics. 2003; 4:2.

94. Hsu SD, Tseng YT, Shrestha S, Lin YL, Khaleel A, Chou $\mathrm{CH}$, Chu CF, Huang HY, Lin CM, Ho SY, Jian TY, Lin FM, Chang TH, et al. miRTarBase update 2014: an information resource for experimentally validated miRNA-target interactions. Nucleic Acids Res. 2014; 42:D78-85.

95. Dweep H, Sticht C, Pandey P, Gretz N. miRWalk-database: prediction of possible miRNA binding sites by "walking" the genes of three genomes. J Biomed Inform. 2011; 44:839-47.

96. Kozomara A, Griffiths-Jones S. miRBase: integrating microRNA annotation and deep-sequencing data. Nucleic Acids Res. 2011; 39:D152-7.

97. Huang DW, Sherman BT, Lempicki RA. Systematic and integrative analysis of large gene lists using DAVID bioinformatics resources. Nature Protoc. 2009; 4:44-57.

98. Chen J, Bardes EE, Aronow BJ, Jegga AG. ToppGene Suite for gene list enrichment analysis and candidate gene prioritization. Nucleic Acids Res. 2009; 37:W305-11.

99. Medina I, Carbonell J, Pulido L, Madeira SC, Goetz S, Conesa A, Tárraga J, Pascual-Montano A, NogalesCadenas R, Santoyo J, García F, Marbà M, Montaner D, et al. Babelomics: an integrative platform for the analysis of transcriptomics, proteomics and genomic data with advanced functional profiling. Nucleic Acids Res. 2010; 38:W210-3. 